\title{
THE ChaMP EXTENDED STELLAR SURVEY (ChESS): PHOTOMETRIC AND SPECTROSCOPIC PROPERTIES OF SERENDIPITOUSLY DETECTED STELLAR X-RAY SOURCES ${ }^{1}$
}

\author{
K. R. Covey,${ }^{2,3,4}$ M. A. Agüeros,${ }^{2,5}$ P. J. Green,${ }^{3}$ D. Haggard,${ }^{6}$ W. A. Barkhouse, ${ }^{7}$ J. Drake, ${ }^{3}$ \\ N. Evans, ${ }^{3}$ V. Kashyap, ${ }^{3}$ D.-W. Kim, ${ }^{3}$ A. Mossman, ${ }^{3}$ D. O. Pease, ${ }^{8}$ and J. D. Silverman ${ }^{9}$ \\ Received 2008 March 16; accepted 2008 May 15
}

\begin{abstract}
We present 348 X-ray-emitting stars identified from correlating the Extended Chandra Multiwavelength Project (ChaMP), a wide-area serendipitous survey based on archival X-ray images, with the Sloan Digital Sky Survey (SDSS). We use morphological star/galaxy separation, matching to an SDSS quasar catalog, an optical color-magnitude cut, and X-ray data-quality tests to create our catalog, the ChaMP Extended Stellar Survey (ChESS), from a sample of 2121 matched ChaMP/SDSS sources. Our cuts retain $92 \%$ of the spectroscopically confirmed stars in the original sample while excluding $99.6 \%$ of the 684 spectroscopically confirmed extragalactic sources. Fewer than $3 \%$ of the sources in our final catalog are previously identified stellar X-ray emitters. For 42 catalog members, spectroscopic classifications are available in the literature. We present new spectral classifications and $\mathrm{H} \alpha$ measurements for an additional 79 stars. The catalog is dominated by main-sequence stars; we estimate the fraction of giants in ChESS is $\sim 10 \%$. We identify seven giant stars (including a possible Cepheid and an RR Lyrae star) as ChaMP sources, as well as three cataclysmic variables. We derive distances from $\sim 10$ to $2000 \mathrm{pc}$ for the stars in our catalog using photometric parallax relations appropriate for dwarfs on the main sequence and calculate their X-ray and bolometric luminosities. These stars lie in a unique space in the $L_{\mathrm{X}}$-distance plane, filling the gap between the nearby stars identified as counterparts to sources in the ROSAT All Sky Survey and the more distant stars detected in deep Chandra and XMM-Newton surveys. For 36 newly identified X-ray-emitting M stars we calculate $L_{\mathrm{H} \alpha} / L_{\mathrm{bol}}$. The quantities $L_{\mathrm{H} \alpha} / L_{\mathrm{bol}}$ and $L_{\mathrm{X}} / L_{\mathrm{bol}}$ are linearly related below $L_{\mathrm{X}} / L_{\text {bol }} \sim 3 \times 10^{-4}$, while $L_{\mathrm{H} \alpha} / L_{\text {bol }}$ appears to turn over at larger $L_{\mathrm{X}} / L_{\text {bol }}$ values. Stars with reliable SDSS photometry have an $\sim 0.1 \mathrm{mag}$ blue excess in $u-g$, likely due to increased chromospheric continuum emission. Photometric metallicity estimates suggest that the sample is evenly split between the young and old disk populations of the Galaxy; the lowest activity sources belong to the old disk population, a clear signature of the decay of magnetic activity with age. Future papers will present analyses of source variability and comparisons of this catalog to models of stellar activity in the Galactic disk.
\end{abstract}

Subject headings: stars: activity — stars: chromospheres — stars: coronae — stars: flare — surveys X-rays: stars

Online material: color figures, machine-readable tables

\section{INTRODUCTION}

While X-ray source counterparts are now known to range from distant quasars to nearby active $M$ dwarfs (e.g., Stocke et al. 1983, 1991; Schmitt et al. 1995; Zickgraf et al. 2003; Green et al. 2004; Anderson et al. 2007), X-ray data alone are frequently insufficient to determine unambiguously whether a given source is Galactic or extragalactic, or to make finer distinctions about its nature. Campaigns to find optical counterparts to X-ray sources have therefore been natural companions to the creation of X-ray source lists since the days of the Einstein Observatory.

The Medium Sensitivity Survey (MSS; Gioia et al. 1984) and Extended Medium-Sensitivity Survey (Gioia et al. 1990) both

\footnotetext{
${ }^{1}$ Observations reported here were obtained at the MMT Observatory, a joint facility of the Smithsonian Institution and the University of Arizona.

2 The first two authors contributed equally to this study.

${ }^{3}$ Harvard-Smithsonian Center for Astrophysics, 60 Garden Street, Cambridge, MA 02138 .

4 Spitzer Fellow.

5 NSF Astronomy and Astrophysics Postdoctoral Fellow; Columbia University, Department of Astronomy, 550 West 120th Street, New York, NY 10027.

${ }^{6}$ NASA Harriett G. Jenkins Predoctoral Fellow; University of Washington, Department of Astronomy, Box 351580, Seattle, WA 98195.

7 Physics Department, University of North Dakota, Grand Forks, ND 58202.

8 Space Sciences Lab, 7 Gauss Way, Berkeley, CA 94720-7450.

9 Max-Planck-Institut für extraterrestrische Physik, D-84571 Garching, Germany.
}

required painstaking programs to identify counterparts to sources serendipitously detected in Einstein observations. To find counterparts to 63 of the 112 MSS sources, Stocke et al. (1983) obtained spectra for all of the optical objects inside or just outside the X-ray $90 \%$ confidence positional error circles: areas of radius $\sim 30^{\prime \prime}$ to $70^{\prime \prime}$. Once they found a plausible counterpart by comparing its $f_{\mathrm{X}} / f_{V}$ to that of similar objects detected in pointed Einstein observations, Stocke et al. (1983) continued to collect spectra until they reached objects at least 4 times fainter than the proposed counterpart or the $\sim 20.5$ mag limit of the Palomar Observatory Sky Survey (POSS). They found that $25 \%$ of MSS sources were coronally emitting stars, primarily late-type dwarfs; they also found one cataclysmic variable $(\mathrm{CV})$.

Similar efforts have been undertaken to identify some of the $\sim 125,000$ sources included in the ROSATAll Sky Survey (RASS) Bright and Faint Source Catalogs (BSC and FSC; Voges et al. 1999). ${ }^{10}$ Only a relatively small fraction of RASS sources can be identified from correlations to existing databases. Bade et al. (1998) found that $35 \%$ of the 80,000 RASS sources they considered had counterparts in SIMBAD and the NASA/IPAC Extragalactic Database. To identify other BSC sources, Bade et al. (1998) used objective-prism spectra obtained as part of the Hamburg Quasar Survey (HQS; Hagen et al. 1995) and found candidate

${ }^{10}$ For FSC, see Vizier Online Data Catalog, 9029 (W. Voges et al. 2000). 
counterparts for $81.2 \%$ of the 3847 sources within the HQS footprint. ${ }^{11}$ One hundred fifty-five (4\%) are M stars, $136(3.5 \%)$ $\mathrm{K}$ stars, and four $(0.1 \%) \mathrm{F}$ or $\mathrm{G}$ stars. Another 956 (24.9\%) are saturated stars $(B \leq 14 \mathrm{mag})$ for which no spectral class is available. There are also 31 white dwarfs (WDs; $0.8 \%$ ) and 16 cataclysmic variables (CVs; $0.4 \%)$. There are uncertainties associated with these identifications, e.g., because of the resolution of the spectra $(R \approx 100$ at $\mathrm{H} \gamma)$. But the RASS/HQS program suggests that $\sim 33 \%$ of the X-ray sources detected by ROSAT are Galactic stars, a result confirmed by later efforts (e.g., Zickgraf et al. 2003).

The Chandra X-ray Observatory and XMM-Newton are both equipped with more sensitive X-ray detectors than ROSAT (albeit in different energy bands) but were designed primarily to conduct pointed observations. However, growing data archives have enabled a number of fairly deep, relatively small-area surveys, with X-ray source lists assembled and optical counterparts identified in much the same way as for the Einstein surveys. In addition, a few deep pencil-beam surveys have been completed with Chandra and XMM-Newton. Brandt \& Hasinger (2005) compare the flux limits and solid angles for a number of these surveys; see their Figure 1.

The selection of optical counterparts for follow-up spectroscopy is generally simpler in these more recent surveys: the X-ray positional uncertainties are very small (typically less than $1^{\prime \prime}$ for Chandra). However, the focus of these surveys is often to characterize faint extragalactic X-ray emitters, and the stellar samples they provide are quite small.

For example, the $X M M$ Bright Serendipitous Survey (BSS; Della Ceca et al. 2004) includes just under 400 sources. The BSS reaches a flux limit of $\sim 7 \times 10^{-14} \mathrm{ergs} \mathrm{cm}^{-2} \mathrm{~s}^{-1}$ in the $0.5-$ $4.5 \mathrm{keV}$ energy band for an area of $28.10 \mathrm{deg}^{2}$. Of the optical counterparts $90 \%$ have magnitudes brighter than the POSS II limit of $R \sim 21 \mathrm{mag}$ (Della Ceca et al. 2004), and close to $90 \%$ of these counterparts now have spectra (López-Santiago et al. 2007). Of these, López-Santiago et al. (2007) identified 58 as stars, which therefore constitute $\sim 15 \%$ of the X-ray counterparts - a smaller fraction than in the Einstein or ROSAT samples but one which is consistent with the positions on the sky of the BSS fields, which are $>20^{\circ}$ from the Galactic plane. These authors compare the colors of their 58 stars to those predicted by the X-ray Galactic model XCOUNT (Favata et al. 1992). They find that model and data agree fairly well for the $\mathrm{M}$ stars in the sample but disagree rather dramatically for F, G, and $\mathrm{K}$ stars. They infer that the discrepancy is due to a stellar population currently absent from their model, possibly known X-ray-emitting binaries such as RS CVn or BY Dra systems.

Feigelson et al. (2004) collected a smaller stellar sample from the Chandra Deep Field-North (CDF-N) survey. The CDF-N has an area of $\sim 448 \operatorname{arcmin}^{2}$; individual exposures were as long as $\sim 2 \times 10^{6} \mathrm{~s}$, resulting in a flux limit of $3 \times 10^{-17} \mathrm{ergs} \mathrm{cm}^{-2} \mathrm{~s}^{-1}$ in the $0.5-2.0 \mathrm{keV}$ band (Alexander et al. 2003). Of the $\sim 500$ sources in the CDF-N, only $\sim 3 \%$ are stars, and Feigelson et al. (2004) use 11 of these to construct a statistically complete sample and study the evolution of X-ray properties. These stars belong primarily to an old disk population (ages between 3 and $11 \mathrm{Gyr}$ ), and their X-ray properties are consistent with a faster than expected decline in magnetic activity $\left(\log L_{\mathrm{X}} \propto t^{-2}\right.$ rather than $t^{-1}$, where $t$ is age; Feigelson et al. 2004).

Studies such as these would clearly benefit from a larger sample of X-ray-emitting stars to analyze. The $X M M$ Slew Survey (Freyberg et al. 2006), constructed from $\leq 15$ s exposures as

\footnotetext{
${ }^{11}$ The unidentified sources are likely to be faint active galactic nuclei and clusters (Bade et al. 1998)
}

the satellite slewed, is one such survey. The recently released XMM-Newton Slew Survey Source Catalog (XMMSL1) covers $\sim 5800 \mathrm{deg}^{2}$ to a relatively shallow flux limit of $6 \times$ $10^{-13} \mathrm{ergs} \mathrm{s}^{-1} \mathrm{~cm}^{-2}$ and includes 2692 sources in its "clean" version (Saxton et al. 2008). A search of the currently available XMMSL1 database finds that $410 X M M$ sources have a star cataloged in SIMBAD within $6^{\prime \prime}$, and it is clear that this program will eventually yield a large number of stellar X-ray sources. However, this stellar sample is still largely undefined. For example, rematching the 410 sources to SIMBAD reveals that $35 \%$ have previously been identified as RASS sources. More work is necessary before we know exactly how many new stellar X-ray sources will come from this survey or the similarly serendipitous 2XMM survey (Watson \& the XMM-Newton Survey Science Centre Consortium 2006).

We have collected from Chandra or $X M M$ data the largest sample of stellar X-ray emitters in the field of the Galaxy identified and characterized to date. The X-ray data are from the Extended Chandra Multiwavelength Project (ChaMP), considerably easing the challenge of identifying the X-ray sources. Chandra provides subarcsecond astrometry over most of its field of view (Aldcroft et al. 2000), greatly facilitating unambiguous matching to optical counterparts, as does the lack of crowding at the high Galactic latitudes of the survey $\left(|b|>20^{\circ}\right)$. In addition, the Extended ChaMP survey is designed to have significant overlap with the Sloan Digital Sky Survey (SDSS), which affords well-calibrated multicolor imaging and spectroscopy crucial both for elimination of extragalactic objects and for classification of stars.

We describe the ChaMP and SDSS in $\S 2$, and the process by which we identify candidate stellar counterparts in $\S 3$. In $\S 4$ we discuss the various tests we use to confirm that these candidates are in fact stellar X-ray emitters. In $\S 5$ we analyze the properties of our resulting sample of $348 \mathrm{X}$-ray-emitting stars; we conclude in $\S 6$. Future work will analyze the X-ray variability of these stars and compare the properties of this catalog to stellar population models of the Galaxy incorporating evolution of time-dependent coronal X-ray emission.

\section{THE SURVEYS}

\subsection{The Extended Chandra Multiwavelength Project}

The Chandra Multiwavelength Project (ChaMP) is a widearea serendipitous survey based on archival X-ray images of the $|b|>20^{\circ}$ sky observed with the Advanced CCD Imaging Spectrometer (ACIS) on board Chandra (described in Weisskopf et al. 2002). The full 130-field Cycle 1-2 X-ray catalogs are public (Kim et al. 2004b, 2007b), and the most comprehensive $\mathrm{X}$-ray number counts $(\log N-\log S)$ to date have been produced, thanks to 6600 sources and massive X-ray source-retrieval simulations (Kim et al. 2004a, 2007a). The simulations added 1000 artificial X-ray point sources across a wide range of fluxes to each actual Chandra ACIS image. The resulting images were subjected to the identical source detection and characterization as used for the actual survey, and a comparison of input and output properties allowed a full calculation of the ChaMP's $\mathrm{X}$-ray sky coverage and completeness as a function of, e.g., source flux and off-axis angle (Kim et al. 2007a).

Green et al. (2004) used deep imaging ( $r \sim 25 \mathrm{mag}$ ) with the National Optical Astronomy Observatory (NOAO) $4 \mathrm{~m}$ telescopes at Kitt Peak National Observatory (KPNO) and Cerro Tololo Interamerican Observatory (CTIO) and follow-up spectroscopy with telescopes ranging from 1.5 to $10 \mathrm{~m}$ in diameter to obtain X-ray source identifications over $14 \mathrm{deg}^{2}$ of the Cycle $1-$ 2 survey. Sixty-six ChaMP fields were imaged in the $g, r$, and 


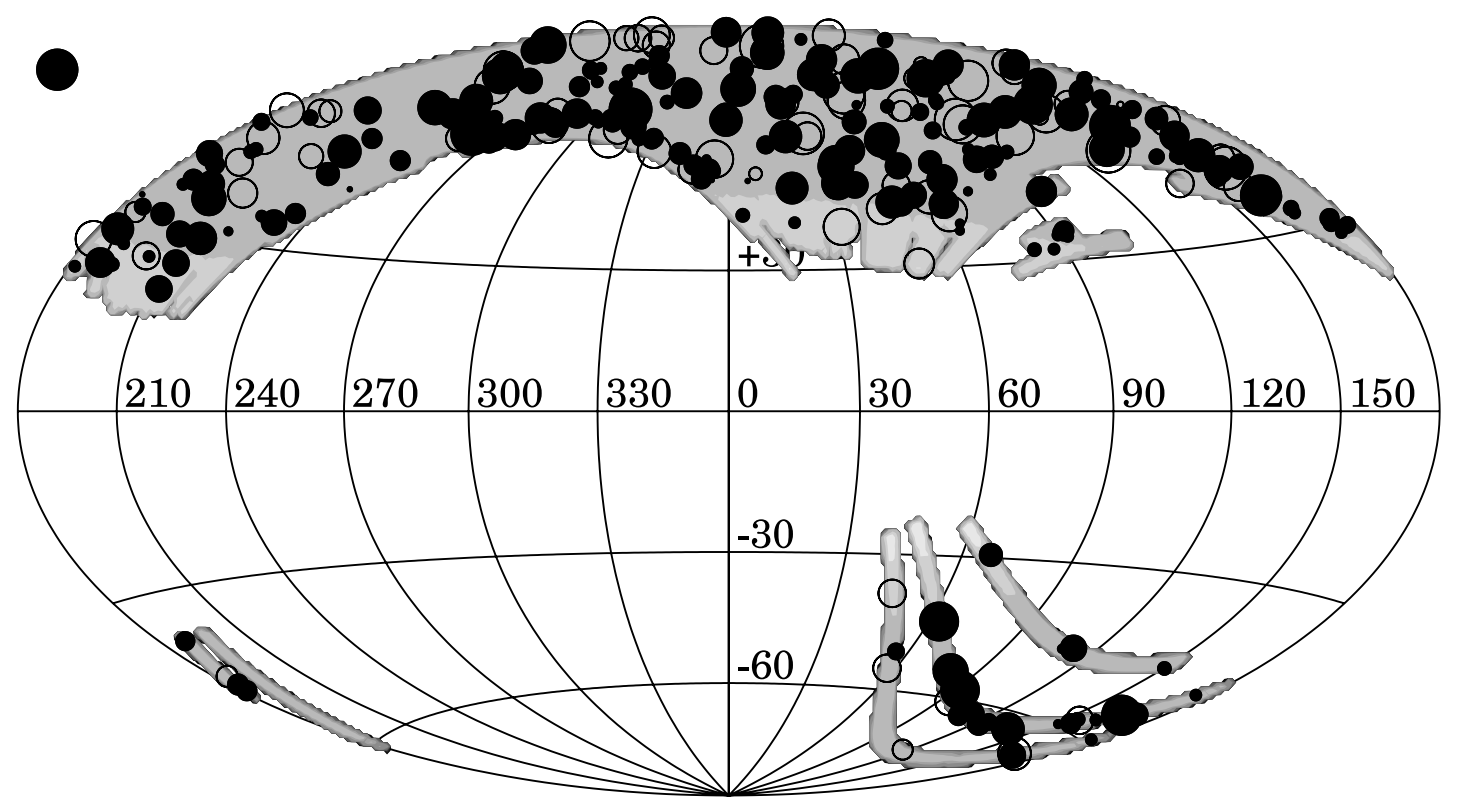

FIG. 1.-Extended ChaMP footprint in Galactic coordinates: open circles, fields observed with the ACIS-I detector; filled circles, fields observed with the ACIS-S detector. The symbol size is proportional to the log of the exposure time; the symbol in the upper left corner corresponds to a $100 \mathrm{ks}$ exposure. The SDSS footprint is the shaded region.

$i$ bands; these data and photometric catalogs are available on the ChaMP Web page ${ }^{12}$ (see also W. A. Barkhouse et al. 2008, in preparation). Optical spectra to $r \sim 22$ were obtained for as many objects as feasible in 27 prime fields, using primarily the WIYN $3.5 \mathrm{~m}$ on Kitt Peak, the MMT with the Blue Channel spectrograph on Mount Hopkins, Arizona, and the Magellan/Baade $6.5 \mathrm{~m}$ telescope with both the LRIS and IMACS spectrographs. A significant number of spectroscopic identifications were also obtained for $r \sim 18$ objects using the Fred Lawrence Whipple Observatory $1.5 \mathrm{~m}$ telescope with the FAST spectrograph. Green et al. (2004) classified 125 X-ray counterparts with optical spectroscopy. Of these, $90 \%$ are extragalactic in nature, as expected (63 are broad-line active galactic nuclei [AGNs]). Silverman et al. (2008) describe the spectroscopic effort in more detail in their paper on the AGN X-ray luminosity function, and a full ChaMP spectroscopic catalog is in preparation.

Given the need for an even wider survey area to accumulate significant samples of rare objects, and the time-consuming nature of deep imaging and spectroscopy, the ChaMP area has been extended to cover archival images from Cycles 1-6 but only to include Chandra images within the SDSS footprint (see $\S$ 2.2). The Extended ChaMP now includes 392 ACIS fields covering a total area of roughly $33 \mathrm{deg}^{2}$ (see Fig. 1) and cata$\operatorname{logs} \sim 17,000 \mathrm{X}$-ray sources. ${ }^{13}$ The median exposure time is $21 \mathrm{ks}$, but individual exposures range from 1 to $119 \mathrm{ks}$. Because of the low Chandra background rates, the formal statistical errors in net counts for each band are consistent within $2 \%$ of Poisson. Here we adopt the more conservative Gehrels (1986) prescription: $\sigma_{\text {cts }}=1+(N+0.75)^{0.5}$.

SDSS photometry within about $20^{\prime}$ of the aim point for each cataloged Chandra observation were obtained to cover the combined ACIS-I and ACIS-S fields of view. ${ }^{14}$ Because the Chandra point-spread function (PSF) increases with off-axis angle, comparatively few $\mathrm{X}$-ray sources are detected beyond this radius, and

\footnotetext{
12 See http://hea-www.harvard.edu/CHAMP/.

13 Some of the weakest sources may be associated with, or contaminated by, cosmic-ray afterglows. Afterglows rarely affect brighter sources or those with bright optical counterparts as in the current sample. See also $\S 4.5$.

${ }^{14}$ For some observations, this was extended to a radius of $28^{\prime}$ to achieve full coverage of the Chandra footprint.
}

source centroids also tend to be highly uncertain. We note that some SDSS imaging strips do not completely cover the Chandra field of view. Detailed X-ray sky coverage versus sensitivity maps represent a major ongoing effort of the ChaMP, described in P. J. Green et al. 2008 (in preparation), which will facilitate accurate volume-limit estimates and allow for, e.g., luminosity-function calculations and stellar-population modeling.

While most ChaMP research to date has emphasized extragalactic objects (e.g., Silverman et al. 2005; Barkhouse et al. 2006; Kim et al. 2006; P. J. Green et al. 2008, in preparation), the ChaMP lends itself well to stellar research. Compared to Galactic plane studies, counterpart identification is very secure at the ChaMP survey's high Galactic latitudes, crowded-field photometry is not an issue, and reddening is quite moderate. In addition, a more balanced ratio of thin/thick-disk populations is sampled. However, the expected fraction of stellar X-ray sources detected in the ChaMP fields is relatively low: ChaMP fields, like those in the $\mathrm{BSS}$, are away from the plane and stars are on average weak X-ray emitters.

\subsection{The Sloan Digital Sky Survey}

The SDSS (Fukugita et al. 1996; Gunn et al. 1998; Hogg et al. 2001; Smith et al. 2002; Gunn et al. 2006) is the deepest largescale optical survey to date and provides uniform photometric (to a depth of $r \sim 22.5$ and an accuracy of $\sim 0.02$ mag; Ivezić et al. 2004) and spectroscopic $(R \sim 1800)$ data sets with which to identify ChaMP sources. The latest data release (DR6; AdelmanMcCarthy et al. 2008) includes imaging for $\sim 9600 \mathrm{deg}^{2}$ and photometry for close to $3 \times 10^{8}$ unique objects. The SDSS spectroscopic footprint is smaller $\left(\sim 7400 \mathrm{deg}^{2}\right)$; spectra over the 3800-9200 $\AA$ range are available for $>10^{6}$ objects. The main spectroscopic samples are for galaxies with Petrosian $r<17.77$ ( $>790,000$ objects) and quasars with PSF $i<19.1$ ( $>100,000$ objects). The DR6 database also includes spectra for close to 300,000 stars, of which nearly 70,000 are of spectral type M or later.

SDSS photometry and spectroscopy has been used to systematically identify RASS sources (e.g., Popesso et al. 2004; Anderson et al. 2007; Parejko et al. 2008; M. Agüeros et al. 2008, in preparation). While the ChaMP is a very different survey from the RASS, 
the SDSS data are equally useful in identifying ChaMP sources, particularly stellar sources. Typical classes of X-ray emitters, including coronally emitting stars, normal galaxies, quasars, and BL Lac objects, have maximum X-ray-to-optical flux ratios corresponding to $\log \left(f_{\mathrm{X}} / f_{\text {opt }}\right)$ values of about $-1,0,+1$, and +1.5 (e.g., Stocke et al. 1991; Zickgraf et al. 2003). Given the typical ChaMP $0.5-2 \mathrm{keV}$ flux ${ }^{15} f_{\mathrm{X}}=10^{-14} \mathrm{ergs} \mathrm{cm}^{-2} \mathrm{~s}^{-1}$, this implies that an optical counterpart for each of these categories of typical X-ray sources will be brighter than 19,21, 24, and 25 mag, respectively. As a result, all but the very faintest stellar optical counterparts to ChaMP sources are bright enough to have confident SDSS photometric detections. Furthermore, such targets may be targeted for SDSS spectroscopy, allowing for secure identifications.

\section{IDENTIFYING CANDIDATE STELLAR SOURCES}

\subsection{Matching to SDSS}

We begin by searching the ChaMP catalog for sources with SDSS counterparts within $20^{\prime \prime}$ of each X-ray source centroid. We identify all potential SDSS matches to a ChaMP source, and we record their distance from the X-ray centroid, along with a ratio of that distance to a radius characterizing the $95 \%$ X-ray position error. The latter depends on both the number of X-ray source counts and the Chandra off-axis angle (Kim et al. 2004b). We then inspect each X-ray source on the smoothed Chandra X-ray image and flag potentially contaminated sources, e.g., those that lie in the outskirts of bright X-ray sources. Detections that appear to be X-ray artifacts are also flagged, but not removed at this stage (see $\S$ 4.5). Using the SDSS Image Tool (Nieto-Santisteban et al. 2004), we simultaneously create SDSS finders for each possible optical match to the X-ray source. Here again, contaminants and potential artifacts (saturation spikes, chip edges, high-background regions, etc.) are noted.

During this visual inspection, a confidence rating is attached to each match from 0 to 3 , with 3 being the highest confidence match. While we flag optically saturated objects during visual inspection, these are not rejected. A match confidence of 3 typically represents a single optical counterpart with a position offset (X-ray to optical) no greater than $2^{\prime \prime}$ and/or less than the $95 \%$ $\mathrm{X}$-ray position error.

We restrict our analysis here to ChaMP sources with a match confidence of 3 and SDSS counterparts with $r<20.5$, a conservative estimate of the faintest magnitude for which SDSS performs robust morphological star/galaxy separation (see $\S 4.2$ ) even under poor observing conditions (Scranton et al. 2002). The resulting catalog contains 2121 ChaMP sources, of which 1320 are classified by SDSS as point sources.

\subsubsection{Estimating the Fraction of Spurious SDSS Matches}

We calculated the separation between the X-ray and optical positions of the 2121 matched objects selected in $\S 3.1$, finding a median X-ray/optical separation of $0.37^{\prime \prime}$ with $\sigma=1.34^{\prime \prime}$. In Figure 2 we show the normalized cumulative histogram of these separations; $90 \%$ of the matched sources have positions in the X-ray and optical catalogs within $3^{\prime \prime}$ of each other.

We then shifted the X-ray source declinations by $+30^{\prime \prime}$ and searched for SDSS matches with $r<20.5$ within $8^{\prime \prime}$ of these new positions, since only 1 of our original 2121 matched objects have separations larger than this. This procedure yields a control sample of 833 matches to these offset X-ray positions.

Figure 2 shows the cumulative normalized histogram for this control sample; as expected, the cumulative fraction rises with

\footnotetext{
15 This flux is the peak of an $f_{\mathrm{X}}$ histogram of ChaMP sources and corresponds approximately to a $50 \%$ completeness limit across the survey.
}

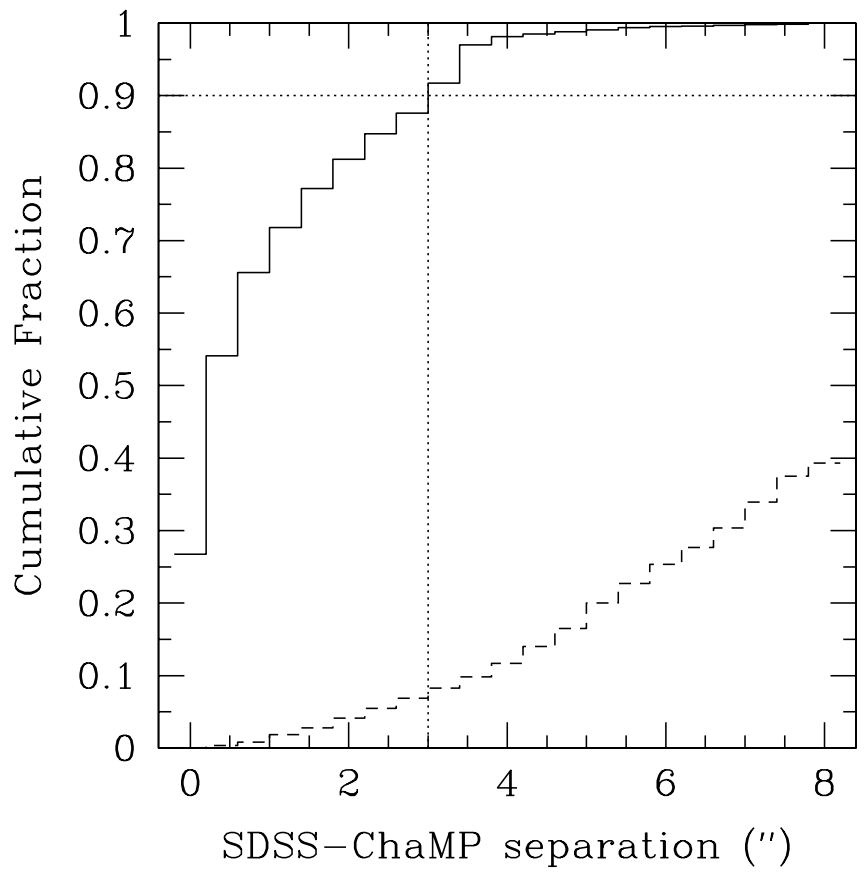

FIG. 2.-Histograms of source separations between ChaMP and SDSS: solid histogram: cumulative distribution of separations between X-ray and optical counterparts for real ChaMP/SDSS sources with $r<20.5 \mathrm{mag}$; dashed line, distribution of separations returned by matching shifted X-ray sources to catalog of SDSS objects with $r<20.5$.

separation. Note that the normalization used here is also 2121, so that the dashed histogram shows an upper limit to the fractional contamination of our sample by chance superpositions of independent X-ray and optical sources. At $3^{\prime \prime}$, the contamination is about $7 \%$. At 4", an X-ray/optical separation larger than or equal to that for $99 \%$ of our sources, the contamination is about $12 \%$. This represents a conservative upper limit, since no SDSS cuts other than $r<20.5$ have been made.

\subsection{Matching to 2MASS}

The Two Micron All Sky Survey (2MASS) obtained nearinfrared images of $99.998 \%$ of the sky between 1997 and 2001 (Skrutskie et al. 1997, 2006; Cutri et al. 2003). The limiting (Vega-based) magnitudes for $10 \sigma$ detections of point sources correspond roughly to $J=15.8, H=15.1$, and $K_{s}=14.3 \mathrm{mag}$. Positional uncertainties are $<0.2^{\prime \prime}$.

We used the Gator interface ${ }^{16}$ to identify 2 MASS counterparts for objects in our catalog, using a $3^{\prime \prime}$ matching radius centered on the X-ray/optical source's SDSS position. For objects with multiple 2MASS sources within $3^{\prime \prime}$, only the closest match was retained. This identified 2MASS counterparts for 889 of the 2121 objects in our initial catalog. We also performed a test similar to that described in $\S 3.1 .1$ to estimate the likelihood of spurious SDSS/2MASS matches by applying a $30^{\prime \prime}$ offset to each source's SDSS position and then identifying 2MASS counterparts within $10^{\prime \prime}$. These false matches tend to have SDSS/2MASS separations of $7^{\prime \prime}-9^{\prime \prime}$, with $90 \%$ lying outside of $3^{\prime \prime}$. The real matches, on the other hand, are all within $3^{\prime \prime} ; 97 \%$ are within $1^{\prime \prime}$.

\section{CONFIRMING THE STELLAR SOURCES}

\subsection{ChaMP Spectroscopy}

We queried the ChaMP spectroscopic database for existing observations and/or classifications of objects in our catalog. All of

16 See http://irsa.ipac.caltech.edu/applications/Gator/. 
the spectra in the ChaMP database have been inspected and visually classified by members of the ChaMP collaboration as either AGN/QSOs, galaxies, or stars. In the ChaMP spectroscopic database 773 sources in our sample have high confidence classifications: of these, 92 have been classified as stellar sources, with the remaining 681 classified as extragalactic and possessing redshifts measured using the IRAF task xcsao. These spectral classifications informed the criteria we develop to remove nonstellar contamination from our sample.

\subsection{SDSS Star/Galaxy Separation}

While SDSS provides automated morphological information for all objects it detects, many of the X-ray sources in our sample have optical counterparts significantly brighter than the SDSS saturation limit $(\sim 15 \mathrm{mag})$. The image flux distribution of saturated stars deviates strongly from a standard PSF, and saturated stars are often classified as extended objects. To ensure accurate morphological classifications, we visually classified the $503 \mathrm{ob}-$ jects with $r<18$. We identified 53 saturated stars misclassified as extended sources by the SDSS pipeline, and we adjusted their entries in our catalog.

We also checked the accuracy of the automated SDSS morphological classification by comparing the spectroscopic and photometric classifications of the 298 morphologically extended objects in our catalog with ChaMP spectra. All but five are classified spectroscopically as extragalactic: 115 are classified as galaxies and 176 as AGN/QSOs. Visual inspection of the SDSS images of these five objects reveals that three (CXOMP J143819.2+ $033349, \mathrm{~J} 112740.4+565309$, and J113311.9+010017) are extended galaxies, suggesting their spectroscopic classification as stars is erroneous. By contrast, CXOMP J142429.9+225641 and J235645.8-010138 are likely stars: they are only marginally resolved and may be either visual binaries or objects with photometric flaws resulting in morphological misclassification.

Of the 298 optically extended objects for which we have spectra, therefore, only two appear to be misclassified stars based on their photometry. This implies that $\lesssim 0.7 \%$ of the objects classified as extended by the SDSS photometric pipeline are actually point sources. Given this, we exclude from further analysis the 748 sources whose optical counterpart has been identified as extended by the pipeline. This increase in sample purity comes at the cost of excluding $\sim 5$ real point sources from our sample, which does not significantly affect our completeness.

Figure 3 presents the 1373 point sources in our initial catalog in various optical and infrared color-color and color-magnitude spaces. Of these point sources 475 have spectroscopic classifications; 87 are identified as stars and 388 as extragalactic in nature. We highlight these two spectroscopic samples in Figure 3.

\subsection{The SDSS Photometric QSO Catalog}

The SDSS provides the largest, most uniform sample of photometrically selected quasars to $i<21$, assembled using a nonparametric Bayesian classification based on kernel density estimation (Richards et al. 2004, 2006, 2007). Each object in the catalog is assigned a photometric redshift according to the empirical algorithm described by Weinstein et al. (2004); the difference between the measured color and the median colors of quasars as a function of redshift is minimized. The quasar catalog utilized in this work includes $\sim 10,000$ SDSS Data Release 5 (DR5; Adelman-McCarthy et al. 2007) photometrically selected QSOs that fall within $20^{\prime}$ of a ChaMP field center (G. Richards 2006, private communication; P. J. Green et al. 2008, in preparation). To minimize QSO contamination, we eliminate from con- sideration the 827 candidate stellar X-ray sources that are listed in the DR5 QSO catalog.

\subsection{A Color-Magnitude Cut}

While matching to the photometrically selected DR5 QSO catalog excludes the vast majority of QSOs in our sample, 47 of the remaining 546 stellar candidates are identified as QSOs in the ChaMP spectroscopic database. As the $g-i$ versus $i$ color-magnitude diagram (CMD) in Figure 3 shows, these QSOs are significantly fainter ( $\geq 2 \mathrm{mag}$ ) than spectroscopically confirmed stars with similar $g-i$ colors. This suggests that a color-magnitude cut can be used to separate stars from QSOs. However, 175 objects still under consideration at this stage are bright enough to saturate pixels in one or more of the five SDSS images, and their SDSSbased colors are untrustworthy.

We therefore restrict our final sample to the 363 sources whose optical counterparts are either flagged as SATURATED in the SDSS database (for a detailed discussion of the SDSS flags, see Stoughton et al. 2002) or are unsaturated and satisfy the $i<$ $16.2+0.7(g-i)$ color-magnitude cut shown in Figure 3. Visual inspection confirms that the 27 objects that are saturated and do not meet our color-magnitude cut are in fact stars.

\subsection{X-Ray Quality Cuts}

We now examine the $\mathrm{X}$-ray properties of the 363 remaining ChaMP sources to identify potential contaminants.

1. Twenty-seven sources are more than $12^{\prime}$ from the Chandra optical axis and are subject to larger photometric and astrometric errors. Since almost all have a large number of counts, we preserve them in our sample. We do flag these sources in our final catalog, however, and we conservatively increase their X-ray flux errors by $50 \%$.

2. Sixteen sources are detected on ACIS S4, which suffers from increased noise and streaking relative to the other Chandra CCDs. These sources are flagged in our final catalog; we conservatively increase their X-ray flux errors by $20 \%$.

3. We find that 14 sources overlap according to the criteria of Kim et al. (2007b). For eight, the overlap is small (as defined by Kim et al. 2007b), and the X-ray photometry is reliable. For the other six, the overlap is large: we flag these sources in our catalog and conservatively double their X-ray flux errors.

4. The exposure times for nine sources are typically less than half the maximum exposure time for their respective CCDs, indicating that the source extraction region encompasses an edge or gap. These sources have unreliable fluxes, and we remove them from our sample.

5. We checked a time-ordered list of photons inside the extraction region for each source in our catalog. We searched for two consecutive photons for which the chip coordinates are the same or differ by 1 pixel, the exposure frames (typically $3.2 \mathrm{~s}$ ) increase by 1 or 2 , and the energies decrease monotonically; these are features associated with cosmic-ray afterglows. ${ }^{17}$ We remove the three false sources (all with $<10$ counts) we found in this manner from our catalog.

In summary, we remove 12 sources from our catalog based on their X-ray properties.

\section{THE ChaMP/SDSS STELLAR CATALOG: ChESS}

Imposing the criteria described above on our initial catalog of 2121 ChaMP detections results in a high-confidence sample of

\footnotetext{
${ }^{17}$ For a description of this problem, see http://asc.harvard.edu/ciao/caveats/ acis_caveats_071213.html..
} 

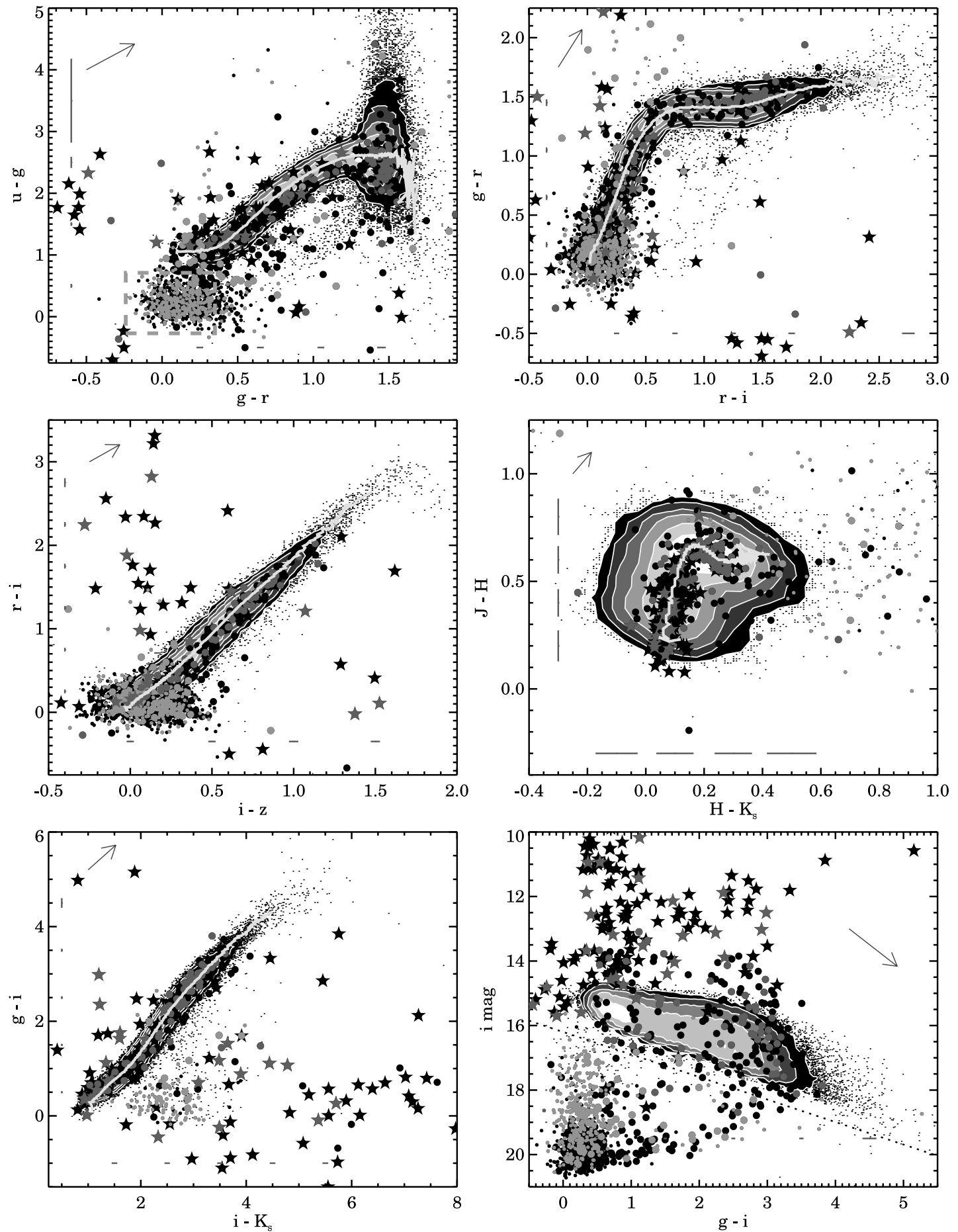

FIG. 3.-Location of our initial catalog in color-color and color-magnitude spaces. All 1373 ChaMP/SDSS point sources are shown as filled symbols, with stars and circles indicating saturated and unsaturated counterparts, respectively. The 87 spectroscopically identified stars are dark gray, while the 388 extragalactic sources are light gray. Objects in the DR5 QSO catalog are shown with half-sized symbols; the box in the top left panel is the area of color space typically inhabited by $z<2.5$ QSOs. Gray-scale contours and black dots show the high-quality sample of SDSS/2MASS point sources presented by Covey et al. (2007); the solid line is the median color-color relation of this sample. The color-magnitude cut described in $\S 4.4$ to eliminate QSOs is shown as a dotted line in the $i$ vs. $g-i$ CMD. Extinction vectors corresponding to $A_{V}=1$ are shown with a arrow in the top left corner of each color-color diagram, and in the upper right of the color-magnitude diagram. The dashed lines along each axis represent the typical photometric errors. [See the electronic edition of the Supplement for a color version of this figure.]

351 stellar X-ray emitters. This sample excludes $99.6 \%$ (681/ 684 ) of the spectroscopically identified extragalactic objects and includes $91 \%(81 / 89)$ of the spectroscopically identified stars. Of the eight spectroscopic stars eliminated from our sample, two lack SDSS counterparts with point-source morphology, one is erroneously listed as having a photometric $z$ in the SDSS QSO catalog, four fail to meet our color-magnitude cut, and one has an
X-ray detection on the edge of a Chandra CCD. We discuss the six eliminated stars with point-source SDSS counterparts in $\S 5.2$.

We remove the three remaining spectroscopically identified QSOs from our sample to produce a final catalog of 348 stellar X-ray emitters, which we define as the ChaMP Extended Stellar Survey (see Table 1 for a summary of the stages in the catalog 
TABLE 1

Stages in Catalog Construction

\begin{tabular}{|c|c|c|c|}
\hline \multirow[b]{2}{*}{$\begin{array}{c}\text { Catalog } \\
\text { (1) }\end{array}$} & \multirow[b]{2}{*}{$\begin{array}{c}\text { Total ObJects } \\
\text { (2) }\end{array}$} & \multicolumn{2}{|c|}{ Spectroscopic } \\
\hline & & $\begin{array}{l}\text { Stars } \\
(3)\end{array}$ & $\begin{array}{l}\text { Galaxies } \\
\text { (4) }\end{array}$ \\
\hline 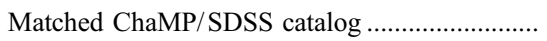 & 2121 & $89(100 \%)$ & $684(100 \%)$ \\
\hline Matched ChaMP/SDSS point sources ................... & 1373 & $87(98 \%)$ & $388(57 \%)$ \\
\hline 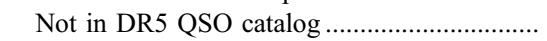 & 546 & $86(97 \%)$ & $47(7 \%)$ \\
\hline With $i<16.2+0.7(g-i)$ & $363^{\mathrm{a}}$ & $82(92 \%)$ & $3(<0.1 \%)$ \\
\hline 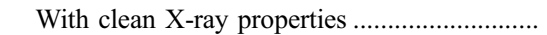 & 351 & $81(91 \%)$ & $3(<0.1 \%)$ \\
\hline 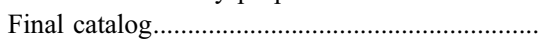 & $348^{\mathrm{b}}$ & $81(91 \%)$ & $0(0 \%)$ \\
\hline
\end{tabular}

Notes.-Columns (3) and (4) give the number of spectroscopically confirmed stars and galaxies, respectively, present in the catalog at each stage. The numbers in parentheses correspond to the fraction of the original number of these objects that is retained.

${ }^{\text {a }}$ Includes 27 saturated stars that do not meet this color-magnitude cut.

b Three spectroscopically confirmed QSOs and 11 sources with substandard X-ray detections are removed manually.

construction). The $348 \mathrm{ChESS}$ stars represent $17 \%$ of the ChaMP sources with SDSS counterparts, a fraction consistent with that found by López-Santiago et al. (2007) as expected. X-ray and optical/near-infrared properties of objects in this catalog are presented in Tables 2 and 3.

\subsection{Previously Cataloged Stars}

A number of ChESS stars are optically bright enough to have been previously cataloged. We search for entries in the SIMBAD catalog within $10^{\prime \prime}$ of the ChESS position for the 348 stars and find that 89 have matches. These stars are discussed in more detail in the Appendix.

The 89 stars can be divided into three groups. The largest group, 66 stars, is made up of optically bright stars that have yet to be identified as X-ray emitters. The first group's natural complement is the small number of stars that have already been identified as $\mathrm{X}$-ray sources; there are only 10 stars for which this is the case. The third group is of ChESS sources included in previous X-ray catalogs but not yet identified; there are 13 such sources. The vast majority of the objects in our catalog, therefore, represent new stellar identifications: previously known stellar X-ray sources make up $<3 \%$ of our sample.

\subsection{Spectroscopic Stellar Sample}

We used the Hammer (Covey et al. 2007), an Interactive Data Language code ${ }^{18}$ to obtain spectral types for the 81 stars in our sample for which we have spectra. The Hammer predicts the Morgan-Keenan (for stars earlier than M) or Kirkpatrick (for later stars) spectral type for a given star on the basis of a fit to a set of 30 spectral indices. In addition, the user can interactively modify the assigned spectral type. Employing this tool, every spectrum was checked by eye and stars were assigned types independently by two authors (M. A. A., K. R. C.). Cases where the types disagreed by more than two subclasses were reexamined. The spectral types ultimately assigned are in Table 4 .

Figure 4 (top) shows the relationship between the assigned spectral types and each star's $g-K_{s}$ color; the close relation between the two quantities (especially for unsaturated stars) suggests that the assigned types are accurate. As an additional test of this accuracy, we plot in Figure 4 (bottom) the difference between the two types initially assigned to each star. The mean difference is slightly more than one subclass, although the quality of

\footnotetext{
18 Available from http://www.cfa.harvard.edu/ kcovey/thehammer.html.
}

the agreement is dependent on the spectral type of the star. The initial independent classifications for $\mathrm{K}$ and $\mathrm{M}$ class stars typically disagree by one subclass or less, while initial classifications for earlier $F$ and $G$ class stars typically disagree by $2-4$ subclasses. We note that while eight of these stars have SIMBAD entries, only three have previously cataloged spectral types and only one is a previously known X-ray emitter. We identify CXOMP J025951.7+ 004619 as [BHR2005] 832-7, which we classify as an M5 star and which SIMBAD lists as an M5.5 V star. CXOMP J122837.1+ 015720 is the known X-ray emitter GSC 00282-00187, classified as an M2 star; we have it as an M1 star. Finally, we identify CXOMP J231820.3+003129 as the F2 star TYC 577-673-1; SIMBAD lists this star as an F5.

We list $\mathrm{H} \alpha$ equivalent widths (EqWs) for each star in Table 4, which we measure by dividing the line flux within a $20 \AA$ window centered at $6563 \AA$ with the continuum flux level determined from a linear fit to two regions (6503-6543 $\AA$ and 6583-6623 $)$. We then use the $\chi$ factor (Walkowicz et al. 2004) to calculate $L_{\mathrm{H} \alpha} / L_{\mathrm{bol}}$ from these EqWs for the $\mathrm{M}$ stars with $\mathrm{H} \alpha$ emission.

As mentioned above ( $\S 4.2$ ), the cuts we use to identify a highconfidence sample of stellar X-ray sources remove five spectroscopically confirmed stars from our catalog. CXOMP J114119.9+ 661006 and J234828.4+005406 are optically faint main-sequence stars with spectral types K7 and M2 and are eliminated by our color-magnitude cut; we remove an M2 star, CXOMP J161958.8+ 292321, because its X-ray detection falls on the edge of a Chandra $\mathrm{CCD}$. The remaining three sources are rarer $\mathrm{CVs}$, which frequently share color space with QSOs:

1. SDSS J020052.2-092431 is a previously unknown CV. Follow-up optical observations are required to determine the nature of the system and its period. Its soft $(0.5-2.0 \mathrm{keV})$ flux is $(3.13 \pm 0.28) \times 10^{-14} \mathrm{ergs} \mathrm{cm}^{-2} \mathrm{~s}^{-1}$, while its broadband $(0.3-$ $8.0 \mathrm{keV}$ ) flux is $(9.04 \pm 0.65) \times 10^{-14} \mathrm{ergs} \mathrm{cm}^{-2} \mathrm{~s}^{-1}$. This CV is eliminated by our color-magnitude cut.

2. SDSS J150722.33+523039.8 was identified as a CV by Szkody et al. (2005). Follow-up photometry revealed that it is an eclipsing system with an extremely short orbital period of only 67 minutes. Furthermore, observations of systems with similarly broad absorption in the Balmer lines suggest that this $\mathrm{CV}$ may contain a pulsating WD (e.g., Woudt et al. 2004). An initial match to the RASS did not return an X-ray counterpart to this CV (Szkody et al. 2005). It was the target of a Chandra observation that is included in the ChaMP database. The CV's soft flux is $(2.36 \pm 0.84) \times 10^{-14} \mathrm{ergs} \mathrm{cm}^{-2} \mathrm{~s}^{-1}$, while its broadband 
TABLE 2

ChaMP Stellar Catalog (X-Rays)

\begin{tabular}{|c|c|c|c|c|c|c|c|c|c|c|}
\hline $\begin{array}{c}\text { Source } \\
\text { (CXOMP) }\end{array}$ & $\begin{array}{l}\text { Chandra } \\
\text { Obs. ID }\end{array}$ & $\begin{array}{c}\mathrm{fBc} \\
\left(10^{-13} \mathrm{ergs} \mathrm{cm}^{-2} \mathrm{~s}^{-1}\right)\end{array}$ & $\begin{array}{l}\text { NetBc } \\
\text { (counts) }\end{array}$ & $\begin{array}{c}\mathrm{fSc} \\
\left(10^{-13} \mathrm{ergs} \mathrm{cm}^{-2} \mathrm{~s}^{-1}\right)\end{array}$ & $\begin{array}{l}\text { NetSc } \\
\text { (counts) }\end{array}$ & $\mathrm{HR}^{\mathrm{a}}$ & $\begin{array}{r}\log \left(L_{\mathrm{Xs}}\right) \\
\left(\mathrm{ergs} \mathrm{s}^{-1}\right)\end{array}$ & $\log \left(L_{\mathrm{X} s} / L_{\mathrm{bol}}\right)$ & $\begin{array}{l}\log \left(L_{\mathrm{X} b}\right) \\
\left(\operatorname{ergs~s}^{-1}\right)\end{array}$ & $\log \left(L_{\mathrm{X} b} / L_{\mathrm{bol}}\right)$ \\
\hline J000155.4+004819 …................ & 4861 & $0.08 \pm 0.08$ & $3.5 \pm 3.4$ & $0.01 \pm 0.03$ & $0.6 \pm 2.3$ & 1.00 & $28.60 \pm 1.30$ & $-4.44 \pm 1.30$ & $29.60 \pm 1.47$ & $-3.45 \pm 1.47$ \\
\hline J001107.9+144153 …….............. & 3957 & $0.19 \pm 0.10$ & $7.7 \pm 4.0$ & $0.10 \pm 0.05$ & $7.9 \pm 4.0$ & -1.00 & $28.34 \pm 0.30$ & $-3.07 \pm 0.30$ & $28.61 \pm 0.31$ & $-2.80 \pm 0.31$ \\
\hline J001313.2+000250 & 4829 & $0.10 \pm 0.05$ & $8.5 \pm 4.1$ & $0.05 \pm 0.02$ & $7.8 \pm 4.0$ & -1.00 & $29.08 \pm 0.31$ & $-4.40 \pm 0.31$ & $29.41 \pm 0.29$ & $-4.07 \pm 0.29$ \\
\hline J003151.4+003233 ….................. & 2101 & $0.59 \pm 0.12$ & $34.7 \pm 7.0$ & $0.29 \pm 0.06$ & $33.9 \pm 6.9$ & -0.90 & $28.27 \pm 0.10$ & $-3.45 \pm 0.10$ & $28.59 \pm 0.10$ & $-3.13 \pm 0.10$ \\
\hline J004238.8-091043 …................ & 4886 & $0.97 \pm 0.13$ & $66.4 \pm 9.2$ & $0.54 \pm 0.08$ & $62.6 \pm 9.0$ & -0.93 & $29.73 \pm 0.07$ & $-3.11 \pm 0.07$ & $29.98 \pm 0.07$ & $-2.86 \pm 0.07$ \\
\hline J010615.6+004814 …................... & 2180 & $0.36 \pm 0.11$ & $16.5 \pm 5.2$ & $0.18 \pm 0.06$ & $15.9 \pm 5.1$ & -0.81 & $28.73 \pm 0.17$ & $-3.31 \pm 0.17$ & $29.03 \pm 0.16$ & $-3.01 \pm 0.16$ \\
\hline J011818.5-005642 …................. & 4963 & $0.14 \pm 0.03$ & $36.0 \pm 8.5$ & $0.09 \pm 0.02$ & $37.5 \pm 7.6$ & -1.00 & $29.25 \pm 0.10$ & $-3.43 \pm 0.10$ & $29.44 \pm 0.12$ & $-3.23 \pm 0.12$ \\
\hline J014821.7+000446 ……............. & 4098 & $0.09 \pm 0.07$ & $4.0 \pm 3.2$ & $0.04 \pm 0.03$ & $3.0 \pm 2.9$ & -0.71 & $28.31 \pm 1.67$ & $-3.11 \pm 1.67$ & $28.73 \pm 0.69$ & $-2.70 \pm 0.69$ \\
\hline J015939.2-084409 ….............. & 6106 & $0.01 \pm 0.02$ & $2.2 \pm 4.3$ & $0.01 \pm 0.01$ & $6.2 \pm 4.0$ & -1.00 & $27.06 \pm 0.44$ & $-5.57 \pm 0.44$ & $26.85 \pm 1.30$ & $-5.79 \pm 1.30$ \\
\hline J015941.6-084506 …................... & 6106 & $0.11 \pm 0.03$ & $31.1 \pm 7.1$ & $0.07 \pm 0.01$ & $30.5 \pm 6.7$ & -1.00 & $27.82 \pm 0.11$ & $-5.11 \pm 0.11$ & $28.05 \pm 0.11$ & $-4.88 \pm 0.11$ \\
\hline
\end{tabular}

Notes.-Table 2 is published in its entirety in the electronic edition of the Astrophysical Journal Supplement. A portion is shown here for guidance regarding its form and content.

Since we do not include any scientific results based on HR, we simply characterize the typical errors here by noting that the mean error on HR is well fit for sources with HR $>-0.98$ by HRerr $=$ $0.36( \pm 0.027) \mathrm{HR}+0.40( \pm 0.022)$, with rms residuals of $\sigma=0.074$. Sources with HR $<-0.98$ have median HR errors of 0.106 , with RMS residuals of $\sigma=0.073$. 
TABLE 3

ChaMP Stellar Catalog (Optical/IR)

\begin{tabular}{|c|c|c|c|c|c|c|c|c|c|c|c|}
\hline Source & $\begin{array}{l}\text { SDSS Sep. } \\
(\operatorname{arcsec})\end{array}$ & $\begin{array}{c}i \\
(\mathrm{mag})\end{array}$ & $\begin{array}{c}u-g \\
(\mathrm{mag})\end{array}$ & $\begin{array}{c}g-r \\
(\mathrm{mag})\end{array}$ & $\begin{array}{c}r-i \\
(\mathrm{mag})\end{array}$ & $\begin{array}{c}i-z \\
(\mathrm{mag})\end{array}$ & $\begin{array}{l}\text { Syn. } g \\
\text { (mag) }\end{array}$ & $\begin{array}{l}\text { Sat. } \\
\text { Flag }\end{array}$ & $\begin{array}{c}J \\
(\mathrm{mag})\end{array}$ & $\begin{array}{l}J-H \\
(\mathrm{mag})\end{array}$ & $\begin{array}{l}H-K_{s} \\
(\mathrm{mag})\end{array}$ \\
\hline J000155.4+004819................ & 5.58 & $14.20 \pm 0.01$ & $1.41 \pm 0.03$ & $0.55 \pm 0.02$ & $0.20 \pm 0.02$ & $0.07 \pm 0.03$ & $15.47 \pm 0.30$ & 1 & $13.24 \pm 0.02$ & $0.38 \pm 0.03$ & $0.07 \pm 0.04$ \\
\hline J001107.9+144153 ................ & 0.72 & $17.16 \pm 0.01$ & $2.02 \pm 0.23$ & $1.51 \pm 0.03$ & $1.63 \pm 0.02$ & $0.89 \pm 0.02$ & $20.49 \pm 0.30$ & 0 & $14.72 \pm 0.03$ & $0.65 \pm 0.05$ & $0.26 \pm 0.07$ \\
\hline $\mathrm{J} 001313.2+000250 \ldots \ldots \ldots \ldots . .$. & 0.47 & $12.56 \pm 0.01$ & $1.17 \pm 0.01$ & $0.31 \pm 0.01$ & $0.10 \pm 0.01$ & $-0.01 \pm 0.03$ & $13.42 \pm 0.30$ & 1 & $11.73 \pm 0.03$ & $0.24 \pm 0.04$ & $0.04 \pm 0.04$ \\
\hline J003151.4+003233 ............... & 0.87 & $14.97 \pm 0.03$ & $2.32 \pm 0.06$ & $1.49 \pm 0.03$ & $1.46 \pm 0.03$ & $0.79 \pm 0.03$ & $17.91 \pm 0.30$ & 0 & $12.70 \pm 0.02$ & $0.56 \pm 0.04$ & $0.28 \pm 0.04$ \\
\hline J004238.8-091043 ............... & 0.24 & $13.36 \pm 0.01$ & $1.85 \pm 0.07$ & $-2.25 \pm 0.07$ & $3.22 \pm 0.02$ & $0.14 \pm 0.02$ & $14.82 \pm 0.30$ & 1 & $12.14 \pm 0.02$ & $0.45 \pm 0.04$ & $0.13 \pm 0.04$ \\
\hline J010615.6+004814................ & 0.23 & $15.75 \pm 0.03$ & $2.48 \pm 0.08$ & $1.40 \pm 0.04$ & $1.24 \pm 0.04$ & $0.67 \pm 0.03$ & $18.26 \pm 0.30$ & 0 & $13.61 \pm 0.03$ & $0.60 \pm 0.04$ & $0.23 \pm 0.04$ \\
\hline J011818.5-005642 …......... & 1.61 & $15.28 \pm 0.02$ & $2.35 \pm 0.04$ & $1.15 \pm 0.04$ & $0.52 \pm 0.03$ & $0.31 \pm 0.03$ & $17.25 \pm 0.30$ & 0 & $13.72 \pm 0.02$ & $0.61 \pm 0.04$ & $0.16 \pm 0.04$ \\
\hline $\mathrm{J} 014821.7+000446 \ldots \ldots \ldots \ldots . .$. & 1.10 & $18.10 \pm 0.01$ & $2.40 \pm 0.80$ & $1.62 \pm 0.05$ & $1.58 \pm 0.03$ & $0.82 \pm 0.03$ & $20.70 \pm 0.30$ & 0 & $15.77 \pm 0.07$ & $0.76 \pm 0.10$ & $0.18 \pm 0.13$ \\
\hline J015939.2-084409 …........ & 1.40 & $15.18 \pm 0.02$ & $2.64 \pm 0.03$ & $-0.96 \pm 0.04$ & $-0.54 \pm 0.04$ & $3.62 \pm 0.02$ & $13.76 \pm 0.30$ & 1 & $10.41 \pm 0.02$ & $0.62 \pm 0.04$ & $0.16 \pm 0.03$ \\
\hline J015941.6-084506 _............. & 0.33 & $10.18 \pm 0.01$ & $1.87 \pm 0.01$ & $0.82 \pm 0.01$ & $0.30 \pm 0.01$ & $-1.97 \pm 0.02$ & $11.82 \pm 0.30$ & 1 & $9.34 \pm 0.02$ & $0.41 \pm 0.06$ & $0.09 \pm 0.06$ \\
\hline
\end{tabular}

Notes.-Table 3 is published in its entirety in the electronic edition of the Astrophysical Journal Supplement. A portion is shown here for guidance regarding its form and content. 
TABLE 4

ChaMP Stars with SPECtra

\begin{tabular}{|c|c|c|c|}
\hline CXOMP & Type & $\begin{array}{c}\mathrm{H} \alpha \mathrm{EqW} \\
(\AA)\end{array}$ & $\log \left(L_{\mathrm{H} \alpha} / L_{\mathrm{bol}}\right)$ \\
\hline 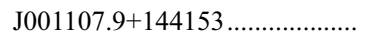 & M5 & 6.09 & -3.78 \\
\hline 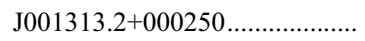 & F7 & -3.67 & . \\
\hline 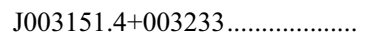 & M4 & 5.01 & -3.50 \\
\hline 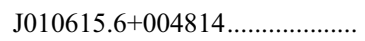 & M3 & 2.71 & -3.70 \\
\hline 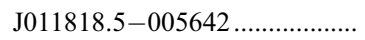 & K5 & 0.71 & $\ldots$ \\
\hline 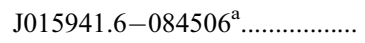 & $\mathrm{K} 2$ & -1.76 & .. \\
\hline J020643.7+121851 …............... & M5 & 6.07 & -3.78 \\
\hline J022429.5-000020 ……............ & M4 & 3.43 & -3.66 \\
\hline J023206.6-073032.. & M4 & 2.75 & -3.76 \\
\hline $\mathrm{J} 025951.7+004619^{\mathrm{b}}$ & M5 & 14.47 & -3.40 \\
\hline $\mathrm{J} 030014.0+004729 \ldots$. & K1 & -1.52 & $\ldots$ \\
\hline $\mathrm{J} 072501.4+371351^{\mathrm{a}} \ldots \ldots \ldots \ldots \ldots \ldots$ & $\mathrm{K} 3$ & -1.15 & $\ldots$ \\
\hline $\mathrm{J} 074108.8+311346^{\mathrm{a}} \ldots \ldots \ldots \ldots \ldots \ldots$ & M6 & 1.24 & -4.66 \\
\hline 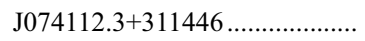 & M4 & 8.60 & -3.26 \\
\hline 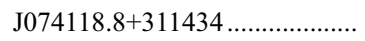 & M3 & 3.53 & -3.58 \\
\hline J074433.8+393027 ..................... & $\mathrm{F} 2$ & -3.55 & $\ldots$ \\
\hline J074437.0+392503 & M5 & 5.71 & -3.81 \\
\hline 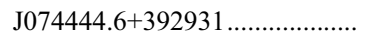 & F5 & -3.69 & $\ldots$ \\
\hline J074705.1+274006 & G1 & -2.53 & $\ldots$ \\
\hline J075549.9+405728 & M2 & +0.09 & -5.08 \\
\hline J075937.3+300846 & G7 & -0.88 & $\ldots$ \\
\hline 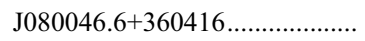 & M2 & -0.05 & $\ldots$ \\
\hline J080048.1+360722 _................... & $\mathrm{F} 1$ & -3.54 & $\ldots$ \\
\hline J080101.0+360549 ...................... & $\mathrm{G} 2$ & -2.57 & $\ldots$ \\
\hline 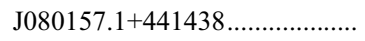 & $\mathrm{K} 2$ & -0.74 & $\ldots$ \\
\hline 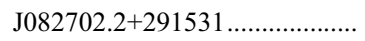 & G4 & -0.94 & $\ldots$ \\
\hline 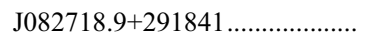 & M0 & -0.45 & $\ldots$ \\
\hline 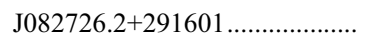 & $\mathrm{G} 2$ & -1.50 & $\ldots$ \\
\hline 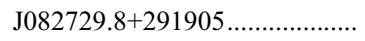 & $\mathrm{K} 2$ & -1.11 & $\ldots$ \\
\hline J082815.2+291132 _.................... & G5 & -2.25 & $\ldots$ \\
\hline J084039.0+130916 ...................... & M0 & -0.30 & $\ldots$ \\
\hline J084044.7+130713 ${ }^{\mathrm{a}} \ldots \ldots \ldots \ldots \ldots$ & G1 & -2.78 & $\ldots$ \\
\hline J084055.8+130800 .................... & M4 & 2.97 & -3.72 \\
\hline J084913.8+444758 ..................... & M0 & -0.42 & $\ldots$ \\
\hline J085325.7+232919 a …................. & M4 & 6.47 & -3.39 \\
\hline J091047.6+541505 ........................ & M2 & 2.62 & -3.60 \\
\hline 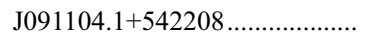 & M2 & -0.52 & $\ldots$ \\
\hline 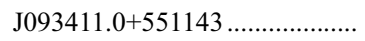 & $\mathrm{F} 4$ & -4.09 & .. \\
\hline J111504.7+403706 _..................... & M3 & 1.04 & -4.12 \\
\hline J111802.3+074325 …................... & G0 & -2.88 & $\ldots$ \\
\hline $\mathrm{J} 112045.7+232536 \ldots \ldots \ldots \ldots \ldots \ldots$ & M2 & 3.67 & -3.45 \\
\hline $\mathrm{J} 112116.2+232622 \ldots \ldots \ldots \ldots \ldots \ldots$ & M3 & 0.17 & -4.90 \\
\hline $\mathrm{J} 114007.3+660659 \ldots \ldots \ldots \ldots \ldots \ldots$ & M2 & 2.43 & $\ldots$ \\
\hline $\mathrm{J} 114101.7+661246 \ldots \ldots \ldots \ldots \ldots \ldots$ & M3 & 0.80 & -4.23 \\
\hline $\mathrm{J} 114129.7+660250 \ldots \ldots \ldots \ldots \ldots \ldots$ & K3 & -1.29 & $\ldots$ \\
\hline J114149.5+661123 _.................... & K7 & -0.31 & $\ldots$ \\
\hline J120439.4-001650 …............... & G8 & -1.98 & $\ldots$ \\
\hline $\mathrm{J} 122155.2+490743 \ldots \ldots \ldots \ldots \ldots \ldots$ & M4 & 4.79 & -3.52 \\
\hline $\mathrm{J} 122738.8+442132 .$. & M4 & 3.28 & -3.68 \\
\hline $\mathrm{J} 122837.1+015720^{\mathrm{b}} \ldots \ldots \ldots \ldots \ldots \ldots$ & M1 & 1.19 & $\ldots$ \\
\hline $\mathrm{J} 125152.2+000528 \ldots \ldots \ldots \ldots \ldots \ldots$ & M3 & 5.23 & -3.41 \\
\hline J131231.0+423106 .................... & M3 & 2.47 & -3.74 \\
\hline J134433.5-000536 ….............. & M4 & 7.23 & -3.34 \\
\hline J134434.8+554956 .................. & M3 & 3.95 & -3.53 \\
\hline J134449.1+555812 ….............. & F8 & -1.81 & .. \\
\hline $\mathrm{J} 134521.5-000118 \ldots \ldots \ldots \ldots \ldots \ldots$ & M3 & 2.43 & -3.75 \\
\hline $\mathrm{J} 140654.3+340949 \ldots \ldots \ldots \ldots \ldots \ldots$ & M4 & 3.81 & -3.62 \\
\hline $\mathrm{J} 141120.7+521411 \ldots \ldots \ldots \ldots \ldots \ldots$ & K7 & -0.94 & $\ldots$ \\
\hline $\mathrm{J} 141715.2+445420 \ldots \ldots \ldots \ldots \ldots \ldots$ & G7 & -1.86 & $\ldots$ \\
\hline $\mathrm{J} 144553.5+012552 \ldots \ldots \ldots \ldots \ldots \ldots$ & M0 & 0.55 & -4.20 \\
\hline 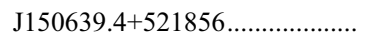 & M6 & 3.43 & -4.22 \\
\hline $\mathrm{J} 151031.7+074248 \ldots \ldots \ldots \ldots \ldots \ldots$ & $\mathrm{K} 2$ & -0.07 & $\ldots$ \\
\hline $\mathrm{J} 151423.8+363511 \ldots \ldots \ldots \ldots \ldots \ldots$ & M3 & 1.35 & -4.00 \\
\hline J153245.2-004012 …............. & G8 & -1.97 & $\ldots$ \\
\hline
\end{tabular}

TABLE 4-Continued

\begin{tabular}{|c|c|c|c|}
\hline CXOMP & Type & $\begin{array}{c}\mathrm{H} \alpha \mathrm{EqW} \\
(\AA)\end{array}$ & $\log \left(L_{\mathrm{H} \alpha} / L_{\mathrm{bol}}\right)$ \\
\hline $\mathrm{J} 153519.7+233152 \ldots$ & M2 & -0.32 & $\ldots$ \\
\hline 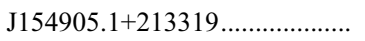 & $\mathrm{K} 3$ & -1.23 & $\ldots$ \\
\hline 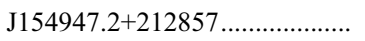 & G3 & -2.57 & $\ldots$ \\
\hline J161958.8+292321 ....................... & M2 & 3.56 & -3.46 \\
\hline 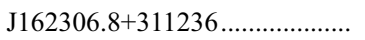 & M4 & 3.85 & -3.61 \\
\hline $\mathrm{J} 162415.4+263728 \ldots \ldots \ldots \ldots \ldots \ldots . .$. & $\mathrm{K} 2$ & -0.90 & $\ldots$ \\
\hline J214218.8+122524_.................... & G2 & -1.72 & $\ldots$ \\
\hline J214229.3+123317 …….............. & M2 & 3.63 & -3.45 \\
\hline J214229.3+123322 ………........... & M4 & 13.54 & -3.06 \\
\hline J214235.6+122701 ……................ & K0 & -1.83 & $\ldots$ \\
\hline J221513.1-004828 ...................... & M2 & 1.39 & -3.87 \\
\hline 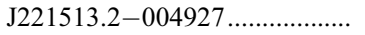 & G4 & -2.77 & .. \\
\hline 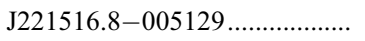 & M4 & 5.51 & -3.46 \\
\hline J221716.9+002208 & K5 & -1.04 & $\ldots$ \\
\hline J221719.1+001428 & K4 & 0.41 & $\ldots$ \\
\hline J224339.9-093348 .................... & $\mathrm{K} 3$ & -0.65 & $\ldots$ \\
\hline J231818.7+003842.. & K7 & -0.96 & $\ldots$ \\
\hline $\mathrm{J} 231820.3+003129^{\mathrm{b}} \ldots \ldots \ldots \ldots \ldots \ldots$ & $\mathrm{F} 2$ & -3.78 & $\ldots$ \\
\hline
\end{tabular}

${ }^{\text {a }}$ Counterpart is cataloged in SIMBAD but lacks a stellar type.

${ }^{\mathrm{b}}$ Counterpart is cataloged in SIMBAD and has a stellar type.

flux is $(7.33 \pm 1.81) \times 10^{-14} \mathrm{ergs} \mathrm{cm}^{-2} \mathrm{~s}^{-1}$. This CV is listed in the SDSS QSO catalog as having a nonzero $z$ and also is eliminated by our color-magnitude cut.

3. SDSS J170053.29+400357.6 is a known X-ray-emitting polar, in which the accretion stream flows directly onto the WD's magnetic poles, with a period of 115 minutes (Szkody et al. 2003). Szkody et al. (2003) convert RASS counts into a flux assuming that for $2 \mathrm{keV}$ bremsstrahlung spectrum, 1 count $\mathrm{s}^{-1}$ corresponds to a $0.1-2.4 \mathrm{keV}$ flux of about $7 \times 10^{12} \mathrm{ergs} \mathrm{cm}^{-2} \mathrm{~s}^{-1}$. In this case, the resulting X-ray flux is $\sim 4.9 \times 10^{-13} \mathrm{ergs} \mathrm{cm}^{-2} \mathrm{~s}^{-1}$. By contrast, the soft Chandra flux is $(2.07 \pm 0.27) \times 10^{-13} \mathrm{ergs} \mathrm{cm}^{-2}$
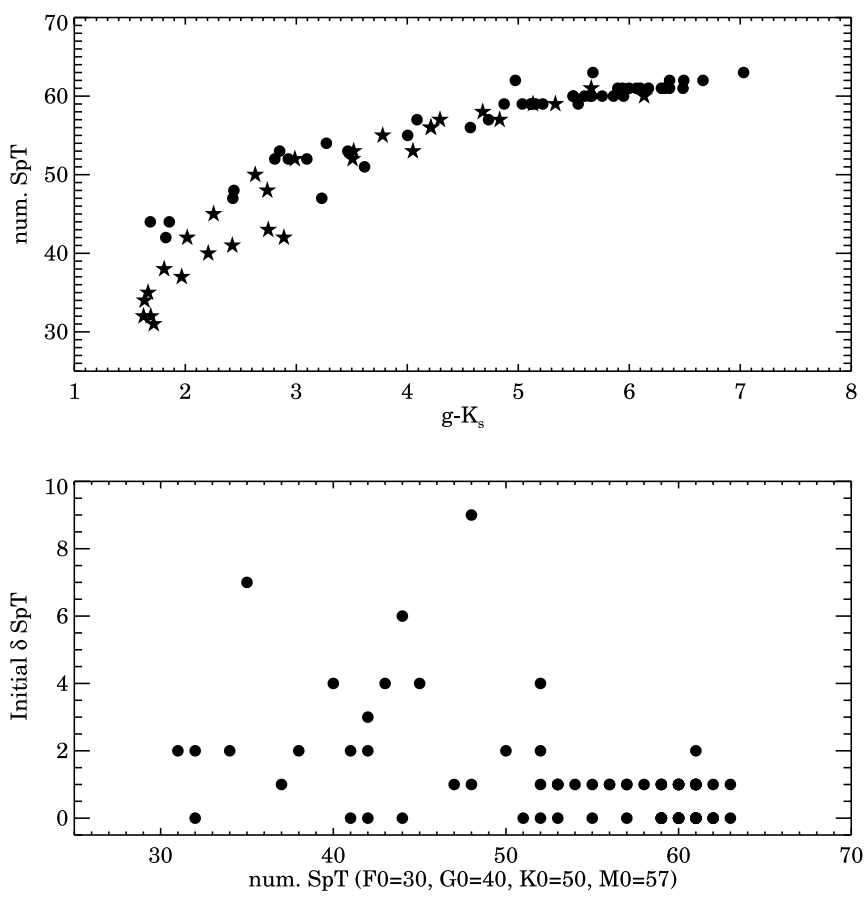

FIG. 4.-Top: Assigned spectral types as a function of $g-K_{s}$; saturated and unsaturated sources are shown as stars and circles, respectively. Bottom: Initial spectral type uncertainty as a function of assigned type. 


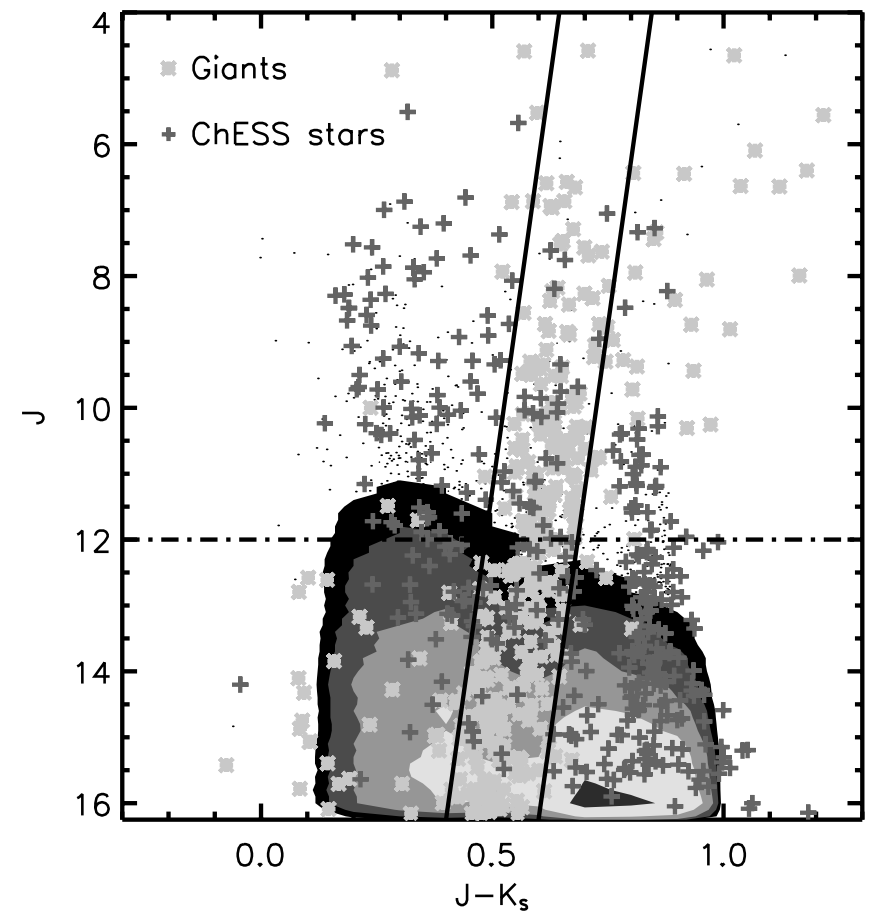

FIG. 5.- Simulated $J$ vs. $J-K_{S}$ color-magnitude diagram, produced by the TRILEGAL galaxy model for SDSS/2MASS observations of a $10 \mathrm{deg}^{2}$ field, with the ChESS stars overplotted (plus signs). The contours and points correspond to the distribution of 10,254 dwarf stars ( $\log g \geq 3.5$ ); 368 giants are highlighted as asterisks. The solid lines enclose the area of the diagram in which giants are most populous. The dot-dashed line is $J=12$; fainter than this magnitude, giants make up only $\sim 10 \%$ of the total number of stars, while brighter than this value they dominate the stellar population. We estimate that $\sim 10 \%$ of the ChESS stars are giants. [See the electronic edition of the Supplement for a color version of this figure.]

$\mathrm{s}^{-1}$, while its broadband flux is $(6.81 \pm 0.62) \times 10^{-13} \mathrm{ergs} \mathrm{cm}^{-2}$ $\mathrm{s}^{-1}$. This $\mathrm{CV}$ is eliminated by our color-magnitude cut.

For all three of these CVs, the broadband flux suggests there is a hard tail to the X-ray emission.

\subsection{Giant Stars}

In order to estimate the fraction of ChESS stars that is likely to be made up of evolved X-ray emitters, we generate simulated SDSS/2MASS observations using the TRILEGAL code (Girardi et al. 2005) and standard Galactic parameters. In Figure 5 we show the resulting $J$ versus $J-K_{s}$ CMD. Dwarf stars are defined as having surface gravities $\log g \geq 3.5$ and their distribution is shown by the density contours and points. The positions of the simulated giant stars are given by asterisks. TRILEGAL predicts that most giants $(78 \%)$ should reside in a fairly narrow locus in $J$ versus $J-K_{s}$ color-magnitude space that stretches from $J \sim 4$ and $0.625 \leq J-K_{s} \leq 0.825$ down to $J \sim 16$ and $0.4 \leq J-$ $K_{s} \leq 0.6$; we highlight this region of the CMD. We then plot the positions of the ChESS stars; 57 inhabit the giant region. However, the relative fraction of giants is not uniform across this region. For stars with $J>12 \mathrm{mag}$, giants represent no more than $11 \%$ of our simulated SDSS/2MASS detections, while they dominate the simulated stellar population at brighter magnitudes. Naively we would therefore only expect 3 of the 29 ChESS stars in the giant region with $J>12$ to be giants; conversely, all 28 ChESS $J<12$ stars in this region are strong giant candidates. Overall, this implies that $\sim 10 \%$ of our sample is made up of giant stars. Our matching to SIMBAD, discussed in $\S 5.1$, identified five known luminosity class III and IV counterparts to ChaMP sources, as well as an RR Lyrae and a candidate Cepheid (see the Appendix), implying that the minimum fraction of ChESS giants is $2 \%$.

\subsection{Stellar Distances}

We wish to derive distances for the ChESS stars using photometric parallax relations appropriate for dwarfs on the main sequence, since these dominate our sample. However, distance estimates based on SDSS photometry are unreliable for the 175 saturated stars in our sample. Fortunately, the SDSS photometric pipeline identifies each object's counterpart in the USNO-B catalog (Monet et al. 2003); similarly, 2MASS uses a $5^{\prime \prime}$ matching radius to identify counterparts in the Tycho 2 or USNO-A2.0 catalogs. As a result, we have either USNO or Tycho counterparts for 347 of the 348 stars in our sample.

We use the Tycho/USNO $B$ magnitudes to construct $B-K_{s}$ colors for each source in the catalog and derive a relationship between $g-K_{s}$ and $B-K_{s}$ for the unsaturated stars:

$$
g-K_{s}=0.93\left(B-K_{s}\right)+0.25
$$

Comparisons of the synthetic $g-K_{s}$ obtained using equation (1) to the measured $g-K_{s}$ for the unsaturated stars reveals that the synthetic $g-K_{s}$ color is accurate to within 0.3 mag $(1 \sigma)$, which we adopt as the characteristic uncertainty for our synthetic $g-K_{s}$.

We then generate synthetic $g-K_{s}$ for the 165 saturated SDSS stars with $B$ magnitudes. We include in Table 3 the synthetic $g$ predicted for each star (calculated from its synthetic $g-K_{s}$ and the observed $K_{s}$ ), as well as a saturation flag that indicates if a star is unsaturated (0), saturated in SDSS with a synthetic $g$ from Tycho/USNO photometry (1), or saturated in SDSS and lacking a Tycho/USNO counterpart (2).

Finally, we use a preliminary fit to the $M_{K_{s}}$ versus $g-K_{s} \mathrm{CMD}$ of D. Golimowski et al. (2008, in preparation), which agrees well with the tabulations of Kraus \& Hillenbrand (2007) to derive distances to each star, using synthetic $g-K_{s}$ colors for stars with saturated SDSS photometry when possible. One star in our sample, CXOMP J153203.5+240501, is undetected in 2MASS, so we estimate its distance using a preliminary fit to the $M_{i}$ versus $g-i$ CMD of D. Golimowski et al. (2008, in preparation).

The resulting distances are shown in Figure 6 as a function of $g-K_{s}$; formal uncertainties in these distances are $<10 \%$, but we adopt conservative uncertainties of $20 \%$ to account for potential systematic errors in the underlying parallax relations. An estimate of the distance limit imposed by the $i$ versus $g-i$ cut described in $\S 4.4$, calculated as a function of $g-K_{s}$ via the color-magnitude data tabulated by Kraus \& Hillenbrand (2007) is shown in Figure 6 as a dashed line. This limit matches the observed upper envelope of the ChESS catalog well. The optical/ near-infrared CMD cut imposes implicit distance limits of between 2000 and $1000 \mathrm{pc}$ for $\mathrm{G}$ and K stars and between 1000 and $200 \mathrm{pc}$ for stars with spectral types M0 to M6.

Five stars in the ChESS catalog have formal distance estimates placing them within $20 \mathrm{pc}$; all five have SIMBAD counterparts. Two, CXOMP J080500.8+103001 and J144232.8+ 011710 , are identified as giant stars, rendering our main-sequence distance estimates invalid. Two others, CXOMP J171954.1+ 263003 and J171952.9+263003, appear to be members of a binary system, despite rather different photometric distance estimates ( 8.2 and $5 \mathrm{pc}$ ); a trigonometric parallax has been derived for the brighter component (J171954.1+263003/V647 Her), placing the system at a distance of $12 \mathrm{pc}$. The last of the five, 


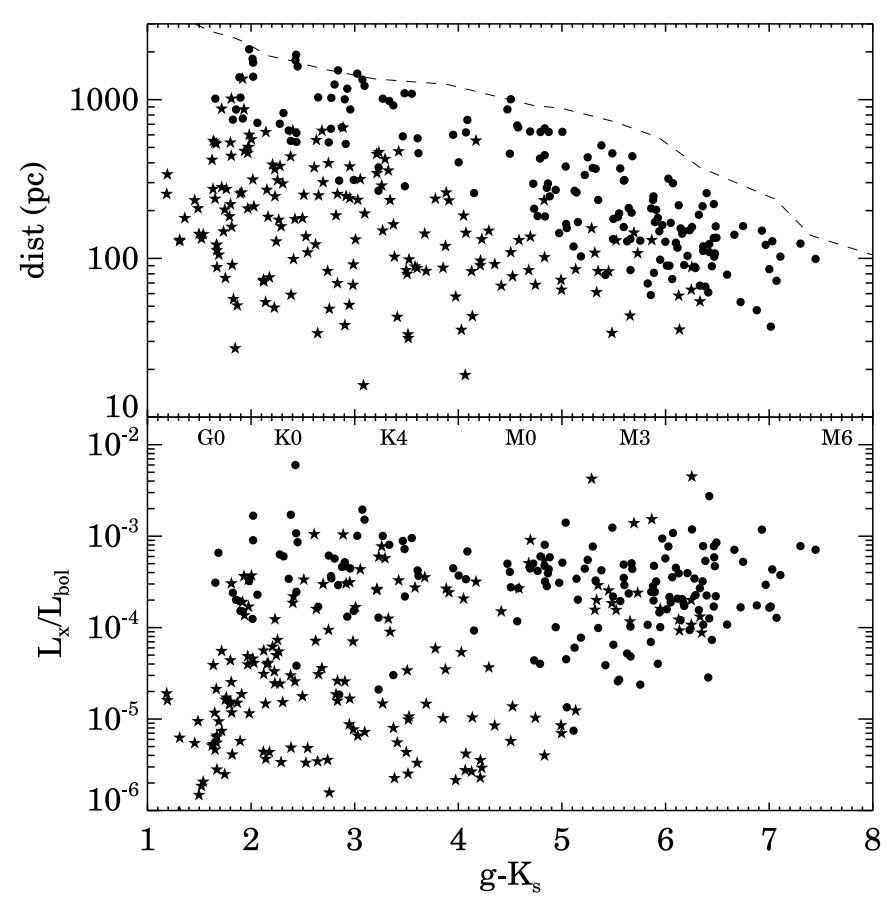

FIG. 6.-Top: Distance to ChESS stars as a function of $g-K_{s}$ color: points, stars with unsaturated SDSS photometry and clean X-ray detections; stars, those with saturated SDSS photometry and/or flagged X-ray detections; dashed line, the distance limit imposed by the $i$ vs. $g-i$ CMD cut described in $\S$ 4.4. Bottom: $L_{\mathrm{X}} / L_{\mathrm{bol}}$ as a function of $g-K_{s}$.

CXOMP J080813.5+210608/LHS 5134, is also likely to be nearby: it is identified in SIMBAD as an M2.5 star, with a distance estimate of $\sim 10 \mathrm{pc}$ from spectroscopic parallax.

\subsection{Stellar X-Ray Luminosities}

Having estimated the distances to our stars, we determine their X-ray luminosities using both the soft $(0.5-2.0 \mathrm{keV})$ and broadband $(0.5-8 \mathrm{keV})$ ChaMP fluxes, whose construction is described in Kim et al. (2007b). ${ }^{19}$ The resulting $L_{X}$ values are included in Table 2; here we limit our discussion to soft X-ray luminosities for comparison purposes. These luminosities are shown in Figure 7 as a function of distance, along with data from several other catalogs of stellar X-ray emitters. The primary source of the comparison data presented here is ROSAT: we include the Schmitt \& Liefke (2004) and Hünsch et al. (1999) catalogs (0.1-2.4 keV luminosities). We also include the 11 stars identified by Feigelson et al. (2004) in the CDF-N (0.5-2 keV) and the nine stars in the LópezSantiago et al. (2007) XMM BSS sample (0.5-4.5 keV) for which they provide distances. Compared to these surveys, the ChESS catalog samples a unique area in the $L_{\mathrm{X}}$-distance plane, covering the ranges of $2 \times 10^{26} \mathrm{ergs} \mathrm{s}^{-1} \lesssim L_{\mathrm{X}} \lesssim 2 \times 10^{31} \mathrm{ergs} \mathrm{s}^{-1}$ and $30 \mathrm{pc} \lesssim d \lesssim 3000 \mathrm{pc}$.

The ChESS stars are for the most part more luminous than those in the volume-complete sample assembled by Schmitt \& Liefke (2004). Despite their low intrinsic luminosities, the nearest stars have moderately large X-ray fluxes $\left(\sim 10^{-12} \mathrm{ergs} \mathrm{cm}^{-2}\right.$ $\mathrm{s}^{-1}$ ). Fields in the Chandra archive including such sources are explicitly excluded from the ChaMP survey: the increased likelihood of saturation in X-ray and optical imaging reduces the ability to detect and classify other X-ray sources in the field and

\footnotetext{
19 Note that this conversion assumes a $\Gamma=1.7$ power-law X-ray spectrum; variations in coronal temperature and metallicity can produce count to fluxconversion factors that differ by a factor of 2 .
}

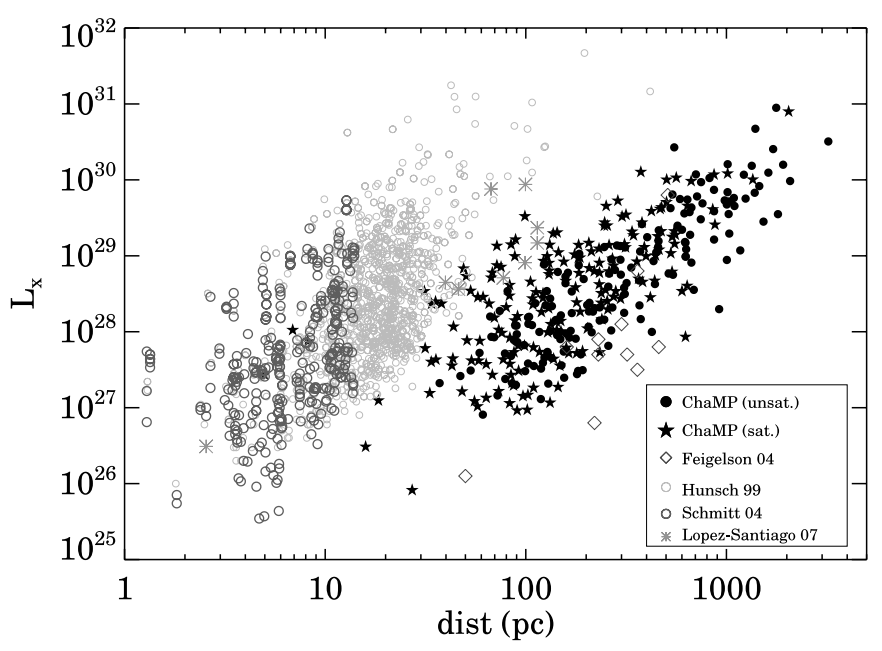

FIG. 7.- Values of $L_{\mathrm{X}}$ as a function of distance for several samples of X-rayemitting stars: filled circles, ChESS stars with unsaturated SDSS photometry and clean X-ray detections; stars, those with saturated SDSS photometry and/or flagged X-ray detections. Also shown are the samples of Schmitt \& Liefke (2004) (open dark gray circles), Hünsch et al. (1999) (open light gray circles), Feigelson et al. (2004) (diamonds), and López-Santiago et al. (2007) (asterisks). [See the electronic edition of the Supplement for a color version of this figure.]

greatly complicates the calculation of the effective area sampled by the observation.

The larger catalog of stellar X-ray emitters assembled by Hünsch et al. (1999) provides a more natural comparison to our ChESS catalog. The $L_{X}$ lower limit of each sample increases with distance, as expected for flux-limited catalogs. While the distance limit of the ChESS catalog is fundamentally optical in nature (due to the CMD cut described in $\S 4.4$ ), a crude comparison of the relative sensitivities of the surveys can be made by comparing the distances to which each instrument can detect stars of a given $L_{X}$ : the Hünsch et al. (1999) sample includes stars with $L_{\mathrm{X}}=10^{28} \mathrm{ergs} \mathrm{s}^{-1}$ to a distance of $30 \mathrm{pc}$, while the ChESS catalog contains such stars out to $200 \mathrm{pc}$. The surface density of stars in the ChESS catalog $\left(\sim 10 \mathrm{deg}^{-2}\right)$ exceeds that of the Hünsch et al. (1999) catalog $\left(3 \times 10^{-4} \mathrm{deg}^{-2}\right)$ by nearly 5 orders of magnitude.

Figure 7 shows that the ChESS stars' properties are most similar to those of stars included in other Chandra and XMM catalogs. These catalogs are not interchangeable, however. For example, while the luminosities of the Feigelson et al. (2004) CDF-N stars are comparable to those of the least luminous members of the ChESS catalog, that sample's effective distance limit is beyond that of the ChESS catalog for equivalent X-ray luminosities. Conversely, because the López-Santiago et al. (2007) sample relies on trigonometric parallax measurements for distances, these $X M M$ detected stars, while also comparably X-ray luminous to the ChESS stars, make up a shallower sample.

We also present in Table 2 the hardness ratio (HR) for each source, where HR $=\left(H_{c}-S_{c}\right) /\left(H_{c}+S_{c}\right)$ and $H_{c}$ and $S_{c}$ are the number of hard and soft counts, respectively (Kim et al. 2007b). The stars in our catalog are quite soft, with typical HRs from -1.0 to -0.6 ; HR shows no clear correlation with $L_{\mathrm{X}}$ or $g-K_{s}$.

\subsection{Stellar Bolometric Luminosities}

For each star, we derive the bolometric luminosity using the $g-K_{s}$ color and the appropriate Kraus \& Hillenbrand (2007) bolometric correction. The resulting $L_{\mathrm{X}} / L_{\text {bol }}$ ratios are presented in Table 2 and shown in Figure 6 (bottom) as a function of $g-K_{s}$. 
The lower limit to the $L_{\mathrm{X}} / L_{\text {bol }}$ values in the ChESS catalog is shaped by the sample's effective $L_{\mathrm{X}}$ limit, which is a function of the exposure times of the Chandra images used to build the ChaMP. The presence of an upper envelope at $L_{\mathrm{X}} / L_{\mathrm{bol}} \sim 10^{-3}$, however, reflects a physical characteristic of the stars. Previous investigators have found a similar empirical upper limit to the efficiency of stellar X-ray emission (e.g., Vilhu \& Rucinski 1983; Vilhu 1987; Herbst \& Miller 1989; Stauffer et al. 1994). While the cause of this so-called saturation is still unknown, it is most commonly attributed to feedback processes that quench the efficiency of the stellar dynamo and/or the ability of the dynamo to heat the coronal plasma (Collier Cameron \& Jianke 1994) or to centrifugal stripping of the coronal plasma at the high rotational velocities associated with large $L_{\mathrm{X}}$ (Jardine 2004).

Figure 8 compares nonsimultaneous measures of the strength of the $\mathrm{H} \alpha$ emission line, a common diagnostic of chromospheric activity, with $L_{\mathrm{X}} / L_{\mathrm{bol}}$, a tracer of coronal activity for stars in our spectroscopic sample. Similar measurements from $\mathrm{M}$ stars in young clusters and the solar neighborhood (e.g., Reid et al. 1995) have found $L_{\mathrm{X}}=(3-5) L_{\mathrm{H} \alpha}$ but were typically made using $R O S A T$ data. As stellar coronae produce very soft X-ray emission, it is unsurprising that the ChESS data, measuring harder X-rays, produces an $L_{\mathrm{X}} / L_{\mathrm{bol}}$ ratio of $\sim 2 / 3$, lower than the ROSAT-measured ratio by a factor of 5 .

The correlation between $L_{\mathrm{X}} / L_{\mathrm{bol}}$ and $L_{\mathrm{H} \alpha} / L_{\mathrm{bol}}$ in the ChESS data, however, is highly significant by Cox proportional hazard $(P=0.0008)$, Kendall's $\tau(P=0.0027)$, and Spearman's $\rho$ tests $(P=0.0064)$, as implemented in the Astronomy Survival Analysis Package (Lavalley et al. 1992). We perform bivariate linear regressions with $\log \left(L_{X} / \mathrm{L}_{\text {bol }}\right)$ as the dependent variable, using the parametric emission measure (EM) algorithm, and find the best-fit relationship

$$
\log \left(L_{\mathrm{H} \alpha} / L_{\mathrm{bol}}\right)=(0.58 \pm 0.13) \log \left(L_{\mathrm{X}} / L_{\mathrm{bol}}\right)-(1.69 \pm 0.48)
$$

with rms residuals of 0.39; this relationship is shown as the solid line in Figure 8. When restricting the sample to $L_{\mathrm{X}} / L_{\mathrm{bol}}<$ $3 \times 10^{-4}$, the best-fit regression line steepens to

$\log \left(L_{\mathrm{H} \alpha} / L_{\mathrm{bol}}\right)=(1.27 \pm 0.24) \log \left(L_{\mathrm{X}} / L_{\mathrm{bol}}\right)+(1.13 \pm 0.94)$,

shown as the dot-dashed line in Figure 8, with rms residuals of 0.31 .

The steepening of the $L_{\mathrm{X}} / L_{\mathrm{bol}}$ versus $L_{\mathrm{H} \alpha} / L_{\mathrm{bol}}$ relation when high $L_{\mathrm{X}} / L_{\mathrm{bol}}$ sources are excluded and the turnover in $L_{\mathrm{H} \alpha} / L_{\mathrm{bol}}$ at large $L_{\mathrm{X}} / L_{\mathrm{bol}}$ that is clearly visible in Figure 8 reveal that stars with very active coronae can possess very pedestrian chromospheres, at least when viewed at distinct epochs. To ensure that this effect is not merely an effect of uncertain $\mathrm{H} \alpha$ measurements in low signal-to-noise ratio $(\mathrm{S} / \mathrm{N})$ spectra, we visually inspected the $\mathrm{H} \alpha$ region in the stars with $L_{\mathrm{X}} / L_{\mathrm{bol}}>3 \times 10^{-4}$. We find that these spectra are of high enough quality to confirm that only very low levels of $\mathrm{H} \alpha$ emission are present in these stars. We also verified that there are no significant differences in the spectral type or Galactic height of stars when the sample is divided at $L_{\mathrm{X}} / L_{\text {bol }}=3 \times 10^{-4}$.

There exist at least two plausible explanations for this seeming disconnect between the chromospheric and coronal properties of the stars with the most active coronae:

1. Our X-ray-selected sample is biased toward detecting flaring stars, whose nonsimultaneous optical spectra may be obtained

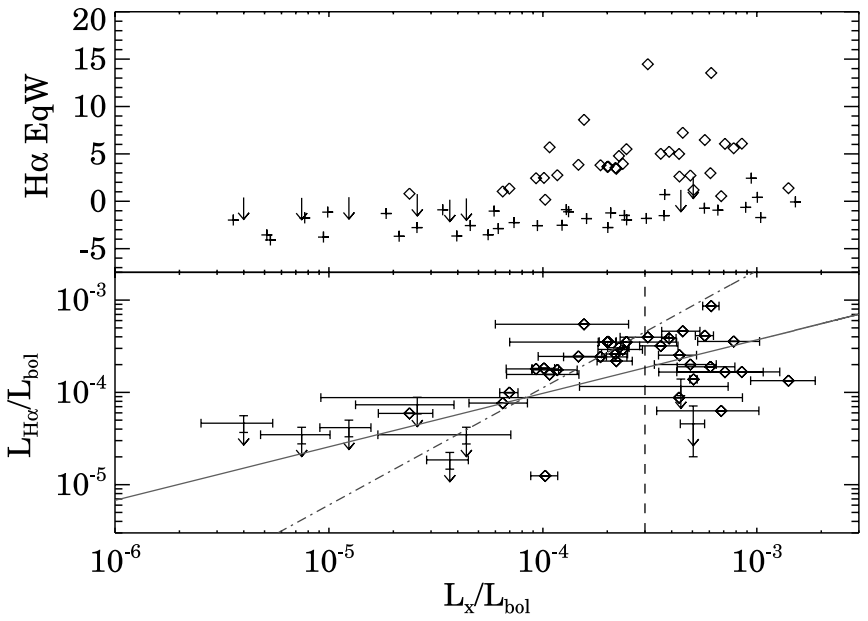

FIG. 8.-Top: $\mathrm{H} \alpha$ EqW vs. $L_{\mathrm{X}} / L_{\text {bol }}$ for stars with ChaMP spectra. Negative EqWs indicate the presence of absorption lines. F, G, and $\mathrm{K}$ stars are shown with plus signs; $M$ stars are indicated with diamonds. The downward-pointing arrows indicate the EqW upper limits for $\mathrm{M}$ stars with no detected $\mathrm{H} \alpha$ emission. Bottom: $L_{\mathrm{H} \alpha} / L_{\text {bol }}$ vs. $L_{\mathrm{X}} / L_{\text {bol }}$ for the $\mathrm{M}$ stars in the spectroscopic sample, with symbols as above. The solid line is the best-fit relation between $L_{\mathrm{H} \alpha} / L_{\mathrm{bol}}$ and $L_{\mathrm{X}} / L_{\mathrm{bol}}$ for the entire sample. The dot-dashed line is the relation for the stars with $L_{\mathrm{X}} / L_{\mathrm{bol}}<3 \times 10^{-4}$, a value indicated by the vertical dashed line. [See the electronic edition of the Supplement for a color version of this figure.]

when the star has returned to quiescence. The seeming disconnect between the coronal and chromospheric properties would then simply reflect the temporal disconnect in the observations of these stars. If this is the case, an extremely crude indicator of the duty cycle of X-ray flares on M stars in the Galactic disk can be derived from the $\sim 45 \%$ (19/43) of the sample with low, and presumably quiescent, $\mathrm{H} \alpha$ luminosity: the failure to observe significant $\mathrm{H} \alpha$ emission during spectroscopic exposures with a median length of $720 \mathrm{~s}$ would imply a upper limit on the typical flare rate of $5 \mathrm{H} \alpha$ flares $\mathrm{hr}^{-1}$.

2. Alternatively, the lack of correlation between chromospheric and coronal emission may be a sign that these two types of activity decouple as coronal activity levels approach the saturated regime. This hypothesis has been advanced previously (e.g., Cram 1982; Pettersen 1987; Mathioudakis \& Doyle 1989; Houdebine et al. 1996); in this scenario, the relative efficiencies of radiative processes that cool the corona and chromosphere (e.g., $\mathrm{H} \alpha, \mathrm{Ca}$ II, and $\mathrm{Mg}$ emission, highly ionized X-ray line emission, and ultraviolet continuum emission) are sensitive to the strength of stellar activity. To explain the effect seen here, extreme levels of stellar activity would have to quench cooling of the chromosphere via $\mathrm{H} \alpha$ emission even as the corona continues to be cooled efficiently by X-rays.

The relatively weak coronae implied by the $L_{\mathrm{X}} / L_{\mathrm{H} \alpha}$ relationship measured from the low-activity portion of our sample and its apparent breakdown at high activity levels present intriguing clues to the temporal behavior of coronal activity over timescales characteristic of both the nonsimultaneity effects $(t<10 \mathrm{yr})$ and population effects $(t>1 \mathrm{Gyr})$ discussed above. The current sample of stars with measurements of both $L_{\mathrm{X}} / L_{\mathrm{bol}}$ and $L_{\mathrm{H} \alpha} / L_{\mathrm{bol}}$ is too small, however, to draw firm conclusions. We defer a full analysis of these effects to follow-up studies.

\subsection{Stellar Colors}

While the clearest signatures of magnetic activity are spectroscopic in nature, stellar activity can impact a star's broadband colors as well. In particular, magnetically active stars appear to possess ultraviolet (UV) excesses of $0.03-0.1 \mathrm{mag}$ in $U-B$ 


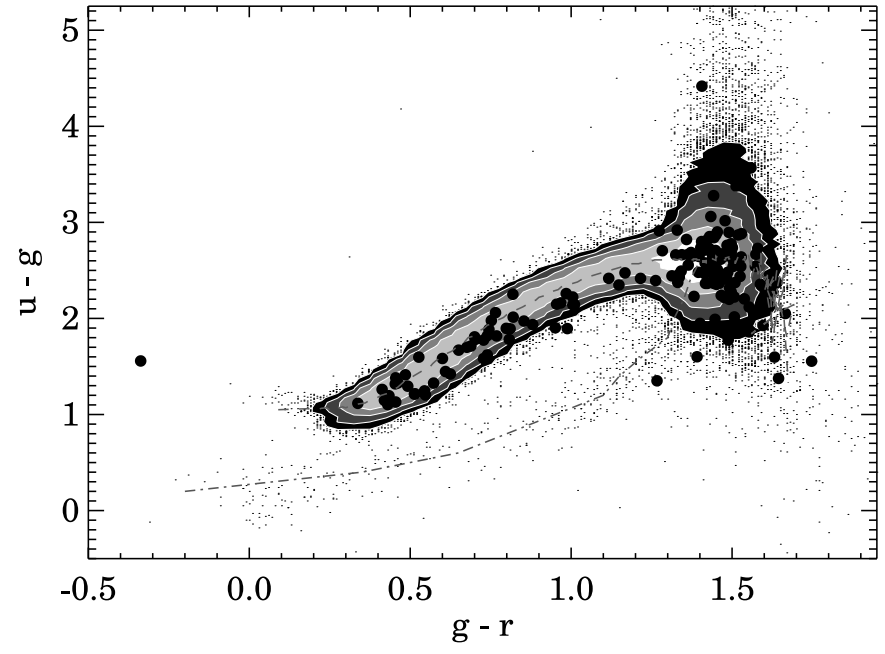

FIG. 9.- Values of $u-g$ vs. $g-r$ for the ChESS stars with unsaturated SDSS photometry and unflagged X-ray detections (filled circles) with the optically selected SDSS /2MASS sample constructed by Covey et al. (2007) shown for comparison, as in Fig. 3. The line running through the contours is the median stellar colors of the Covey et al. (2007) sample; the lower, dot-dashed line shows the locus of WD/M dwarf pairs identified by Smolčić et al. (2004). [See the electronic edition of the Supplement for a color version of this figure.]

compared to nonactive stars. This excess has been attributed to continuum emission generated from hot, active chromospheres (Houdebine et al. 1996; Houdebine \& Stempels 1997; Amado \& Byrne 1997; James et al. 2000; Sung et al. 2002; Amado 2003; Bochanski et al. 2007).

The $u-g$ versus $g-r$ color-color diagram in Figure 9 shows evidence for a similar shift, with X-ray-emitting, optically unsaturated ChESS stars lying systematically lower than the median $u-g$ versus $g-r$ locus measured by Covey et al. (2007) from a sample of optically selected SDSS/2MASS stars. This shift in color-color space, however, is not unambiguous proof of a $u-g$ excess, as the offset could be caused by a red excess in $g-r$, particularly since active stars can have strong $\mathrm{H} \alpha$ emission that contributes additional flux to the $r$ band. Our spectroscopic sample, however, does not include any stars with $\mathrm{H} \alpha$ equivalent widths significantly larger than $10 \AA$ (see Table 4), and even such strong $\mathrm{H} \alpha$ emission lines contribute only a small fraction to the flux transmitted through $\mathrm{a} \sim 1000 \AA$ wide filter, brightening a star in the $r$ band by only $0.01 \mathrm{mag}$.

To confirm that the offset in $u-g$ versus $g-r$ is due to the stars' anomalous $u-g$ colors, we compare the offsets between the $u-g$ and $g-r$ colors of unsaturated stars in our sample and the median colors of nonactive stars with the same $i-K_{s}$ color tabulated by Covey et al. (2007) (see Fig. 10, top). While the spread is large, active stars are systematically bluer by 0.12 mag in $u-g$ than inactive stars. By contrast, the $g-r$ colors of active stars are consistent with those of inactive stars to within $0.03 \mathrm{mag}$, and there the difference is that active stars are bluer than inactive stars. This is inconsistent with the idea of a redshift caused by the addition of $\mathrm{H} \alpha$ emission into a star's $r$ band.

While stellar $u-g$ colors are sensitive to metallicity and the presence of unresolved WD companions, neither effect is likely to explain the offset seen here. The sensitivity of $u-g$ to metallicity is due to line blanketing, where absorption by a large number of metal lines in the $u$ band leads to preferentially redder $u-g$ colors for more metal-rich stars. Interpreted as a metallicity effect, however, the $\sim 0.1$ mag blue $u-g$ offset implies that $\mathrm{X}$-ray luminous stars have metallicities more than half a dex lower than the standard field population (Karaali et al. 2005), exceed- ingly unlikely given the well-known link between stellar age and X-ray luminosity.

Similarly, while main-sequence stars with an unresolved WD companion have anomalously blue $u-g$ colors, as well as the potential for enhanced X-ray luminosity, the colors of the stars in our sample disagree with those expected for such binaries. The SDSS colors of WD/main sequence binaries found by Smolčić et al. (2004) and Silvestri et al. (2006) are shown in Figure 9. While there may be a handful of such systems in our sample, the bulk of the ChESS stars are redder in $u-g$ than would be expected for systems with WD components.

To investigate the cause of this $u-g$ offset, Figure 10 also shows the magnitude of the $u-g$ offset as a function of $L_{\mathrm{X}}, L_{\mathrm{X}} /$ $L_{\mathrm{bol}}$, and $i-K_{s}$, a proxy for stellar temperature and mass. A slight tendency for the offset to increase with $L_{\mathrm{X}} / L_{\mathrm{bol}}$ may be present, particularly when considering only stars of a given spectral type, but linear regression does not return a statistically significant correlation between the two variables. One would expect the $u-g$ excess to be most prominent for M stars, which typically have the highest activity and the lowest quiescent UV flux, allowing contributions from the chromosphere to affect the stars' $u-g$ most significantly. Instead, the $u-g$ excess reaches a maximum for $\mathrm{K}$ stars (at $i-K_{s} \sim 2.0$ ) and then decreases into the $\mathrm{M}$ regime. Whether this effect is real or the result of observational bias is hard to access, in part because of the increased uncertainties in $u-g$ for late-type stars caused by the red leak in the SDSS camera. ${ }^{20}$ The additional scatter in the $u-g$ colors of these stars may wash out evidence for trends of $\delta(u-g)$ with either $L_{X}$ or color. Follow-up studies with more reliable $u$ photometry are needed to reveal the nature of any correlation between $u-g$ excess and coronal or chromospheric activity.

\subsection{Stellar Populations}

Previous studies have found that magnetically active stars have a smaller Galactic scale height than nonactive stars (e.g., West et al. 2008). To determine how the stars in our catalog are distributed between the different Galaxy components, we use each star's distance and Galactic latitude to calculate its height in the Galactic disk. We show in Figure 11 the resulting Galactic heights as a function of both Galactic latitude and stellar color. If our catalog were probing a spherically symmetric halo population, the color-magnitude cut imposed in $\S 4.4$ would limit the catalog mainly as a function of the heliocentric distance to each star. Sight lines probing higher Galactic latitudes would sample stars at larger Galactic heights. The distribution of Galactic heights in the sample is independent of Galactic latitude, however, indicating that the distribution of stars within the disk of the Milky Way imposes a stricter distance limit than the color-magnitude cut imposed in $\S 4.4$.

Stauffer \& Hartmann (1986) and Leggett (1992) have correlated the near-infrared colors of M stars and their metallicities and kinematics, allowing them to define regions of $J-H$ versus $H-K_{s}$ color-color space dominated by young and old disk stars. In Figure 12 (top), we compare the $J H K_{s}$ colors of M stars in our sample to the boundary defined by Leggett (1992) between young

\footnotetext{
20 The red leak describes an instrumental effect whereby the $u$-band filter transmits flux longward of $7100 \AA$ due to changes in the filter's interference coating under vacuum. This instrumental effect depends on a star's raw $u$ and $r$ magnitudes, which in turn are dependent on the air mass, seeing, and the sensitivity of each $u$ filter as a function of wavelength and stellar spectrum. Given the complexity of this effect, the SDSS photometric pipeline does not attempt to correct each star's $u$-band photometry, resulting in increased $u$ uncertainties of $0.02 \mathrm{mag}$ for $\mathrm{K}$ stars, $0.06 \mathrm{mag}$ for M0 stars, and $0.3 \mathrm{mag}$ for stars with $r-i>1.5$. For more information see http://www.sdss.org/dr6/products/catalogs.
} 

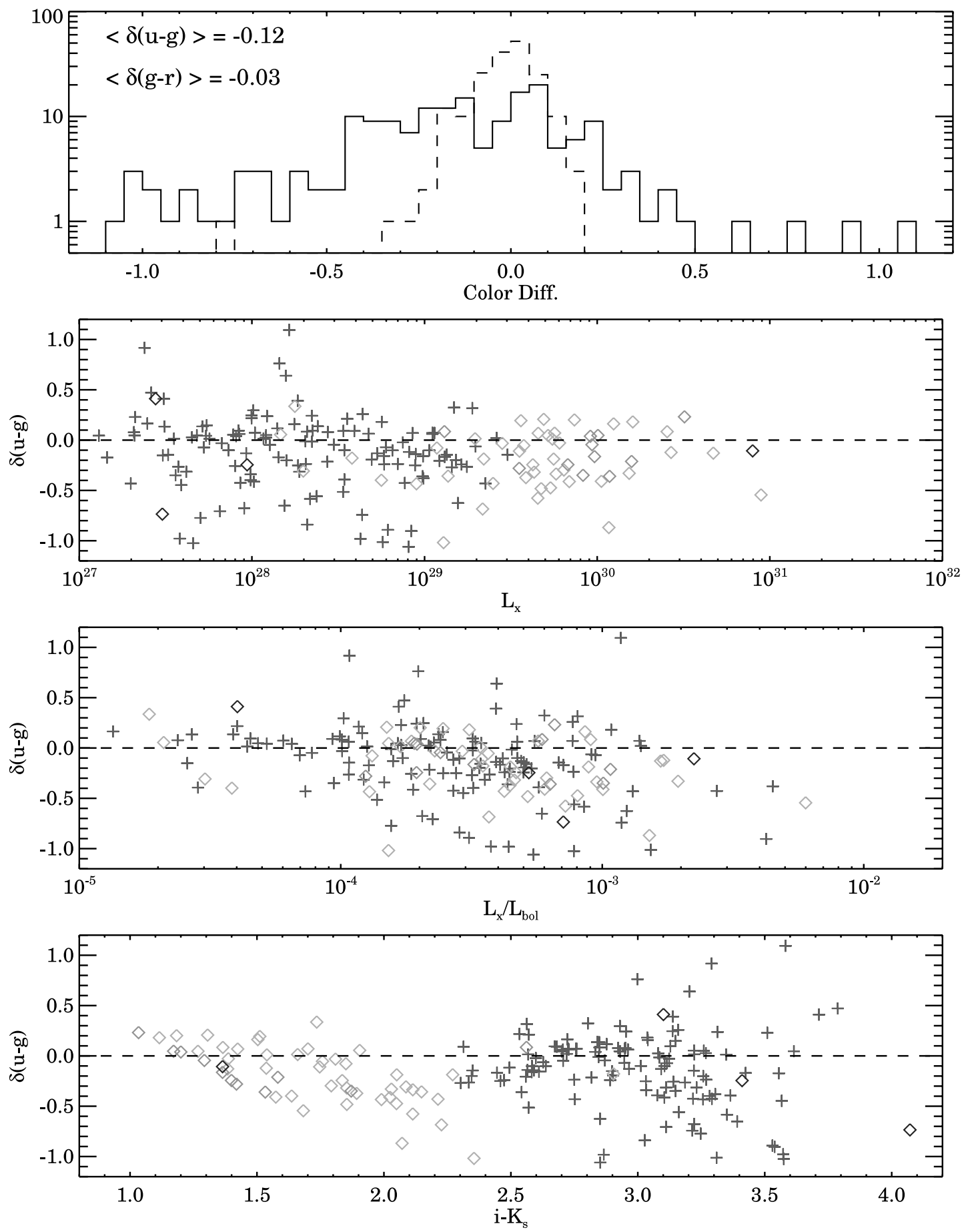

FIg. 10.-Top: Histograms of color differences between unsaturated ChESS stars and optically selected stars with identical $i-K_{s}$. Differences for $u-g$ (solid line) and $g-r$ (dashed line) are shown. Second from top: $u-g$ differences for unsaturated stars as a function of $L_{\mathrm{X}}$. M stars are shown as crosses, while $\mathrm{F}, \mathrm{G}$, and $\mathrm{K}$ stars are shown as diamonds; lighter diamonds indicate later-type stars. Third from top: $u-g$ differences for unsaturated stars as a function of $L_{\mathrm{X}} / L_{\mathrm{bol}}$. Bottom: $u-g$ differences for unsaturated stars as a function of $i-K_{s}$. [See the electronic edition of the Supplement for a color version of this figure.]

and old disk stars. This boundary nearly bisects our sample, suggesting that the ChESS catalog contains both young stars and the high-activity tail of the old disk population. Figure 12 (bottom) also shows $L_{\mathrm{X}} / L_{\text {bol }}$ for these M stars as a function of their offset from the young/old-disk boundary. The lowest activity sources $\left(L_{\mathrm{X}} / L_{\text {bol }} \sim 10^{-5}\right.$ ) are uniformly identified with the old disk population, a clear signature of the decay of magnetic activity with age. Interpreting the significance of the many old disk stars with large $L_{\mathrm{X}} / L_{\text {bol }}$ values is less straightforward, particularly because these active old disk stars are likely merely color outliers of the vastly more numerous young disk population. If these high $L_{\mathrm{X}} /$ $L_{\text {bol }}$ stars are truly members of the old disk, however, they would represent a new and very significant population of stars that experience little decay of magnetic activity over their lifetimes.

\section{CONCLUSIONS}

We have correlated the Extended Chandra Multiwavelength Project with the Sloan Digital Sky Survey to identify the 348 X-ray-emitting stars of the ChaMP Extended Stellar Survey. We used morphological star/galaxy separation, matching to an 


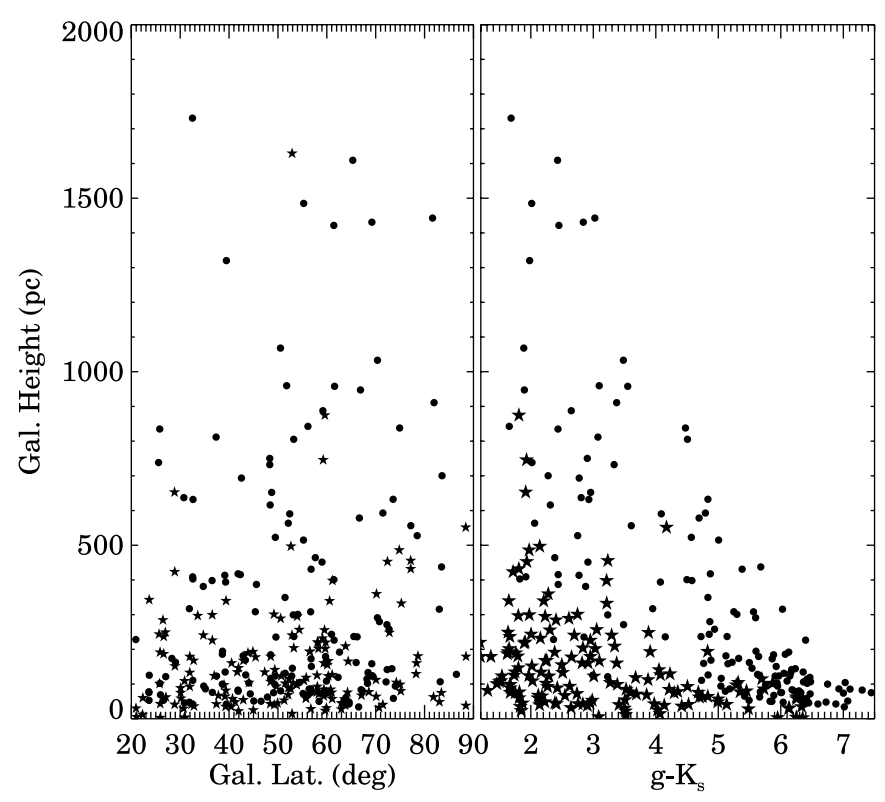

FIG. 11.-Left: Height in the Galactic disk (in parsecs) as a function of Galactic latitude. Right: Height in the Galactic disk (in parsecs) as a function of $g-K_{s}$. Symbols are as in Fig. 7.

SDSS quasar catalog, an optical color-magnitude cut, and X-ray data-quality tests to identify the ChESS stars from a sample of 2121 matched ChaMP/SDSS sources.

1. Our cuts retain $91 \%$ of the spectroscopically confirmed stars in the original sample while excluding $99.6 \%$ of the 684 spectroscopically confirmed extragalactic sources. Fewer than $3 \%$ of the sources in our final catalog are previously identified stellar $\mathrm{X}$-ray emitters.

2. For 42 catalog members, spectroscopic classifications are available in the literature. We present new spectral classifications and $\mathrm{H} \alpha$ measurements for an additional 79 stars. We derive distances to the stars in our catalog using photometric parallax relations appropriate for dwarfs on the main sequence and calculate their X-ray and bolometric luminosities. For 36 newly identified X-ray -emitting $\mathrm{M}$ stars we also provide measurements of $L_{\mathrm{H} \alpha} / L_{\mathrm{bol}}$.

3. The stars in our catalog lie in a unique space in the $L_{X^{-}}$ distance plane, filling the gap between the nearby stars identified as counterparts to sources in the ROSAT All Sky Survey and the more distant stars detected in other Chandra and XMM-Newton surveys.

4. The ChESS catalog is dominated by main-sequence stars. By comparing the distribution of the ChESS sample in $J$ versus $J-K_{S}$ space to that of simulated SDSS/2MASS observations generated by TRILEGAL, we estimate that the total fraction of giants in the catalog is $\sim 10 \%$. In addition to seven confirmed giant stars (including a possible Cepheid and an RR Lyrae star), we identify three cataclysmic variables.

5. We find that $L_{\mathrm{H} \alpha} / L_{\mathrm{bol}}$ and $L_{\mathrm{X}} / L_{\mathrm{bol}}$ are linearly related below $L_{\mathrm{X}} / L_{\text {bol }} \sim 3 \times 10^{-4}$, while $L_{\mathrm{H} \alpha} / L_{\text {bol }}$ appears to turn over at larger $L_{\mathrm{X}} / L_{\mathrm{bol}}$ values.

6. Stars with reliable SDSS photometry have an $\sim 0.1 \mathrm{mag}$ blue excess in $u-g$, likely due to increased chromospheric continuum emission. Photometric metallicity estimates suggest that our sample is evenly split between the young and old disk populations of the Galaxy; the lowest activity sources are identified with the old disk population, a clear signature of the decay of magnetic activity with age.
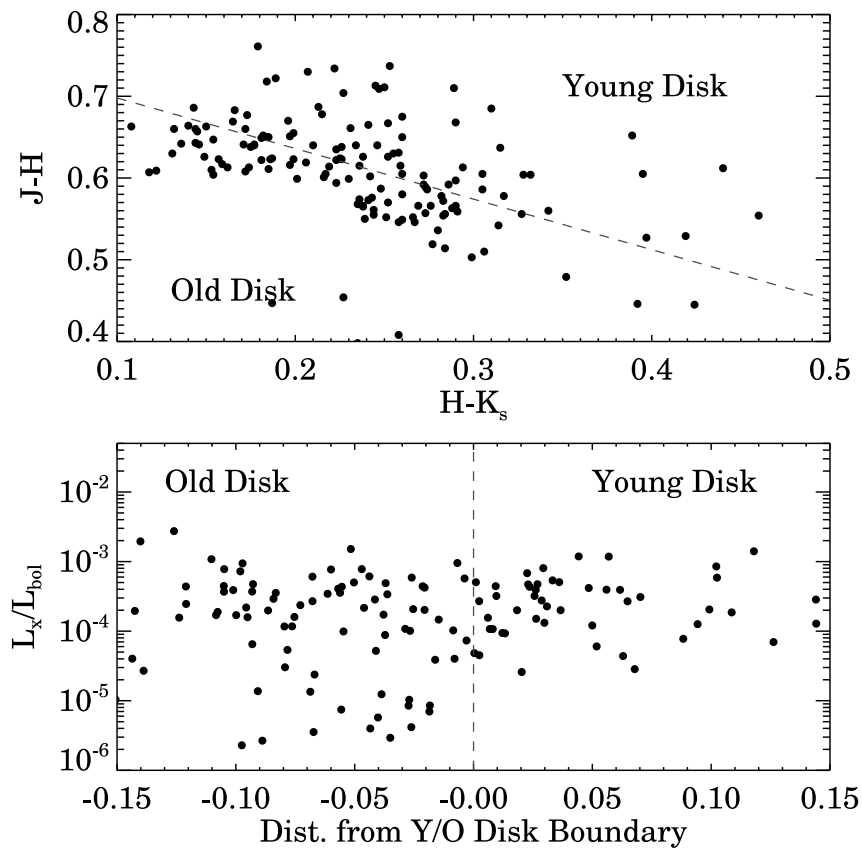

FIG. 12.-Top: Values of $J-H$ vs. $H-K_{s}$ for ChESS M stars with unflagged $\mathrm{X}$-ray detections. The dashed line is the boundary between the regions identified by Stauffer \& Hartmann (1986) and Leggett (1992) as populated preferentially on one side by relatively high-metallicity young-disk stars and on the other by relatively low-metallicity old-disk stars. Bottom: $L_{\mathrm{X}} / L_{\mathrm{bol}}$ as a function of offset in $J-H$ from the young-disk/old-disk boundary in the top panel.

Future papers will present analyses of ChESS source variability and comparisons of the ChESS catalog to models of stellar activity in the Galactic disk.

We thank Suzanne Hawley, Andrew West, Steven Saar, and Thomas Fleming for useful discussions of stellar magnetic activity; we also thank the anonymous referee and editor for useful comments that improved the work presented here. We are indebted to the staffs at the National Optical Astronomy Observatories (NOAO), Las Campanas, and the MMT for assistance with optical spectroscopy. Special thanks to observers including Warren Brown, Perry Berlind, and Michael Calkins, for FAST spectroscopy from the Fred Lawrence Whipple Observatory $1.5 \mathrm{~m}$ on Mount Hopkins, and to Susan Tokarz and Nathalie Marthimbeau for reductions.

Support for this work was provided by the National Aeronautics and Space Administration (NASA) through Chandra, award AR4-5017X and AR6-7020X issued by the Chandra X-ray Observatory Center, which is operated by the Smithsonian Astrophysical Observatory for and on behalf of NASA under contract NAS 08-03060. Further NASA support was provided to K. R. C. through the Spitzer Space Telescope Fellowship Program, through a contract issued by the Jet Propulsion Laboratory, California Institute of Technology under a contract with NASA. M. A. is supported by an National Science Foundation (NSF) Astronomy and Astrophysics Postdoctoral Fellowship under award AST 0602099. D. H. is supported by a NASA Harriett G. Jenkins Predoctoral Fellowship.

This work is based in part on observations obtained at CTIO and KPNO, NOAO, operated by the Association of Universities for Research in Astronomy, Inc. under cooperative agreement with the NSF. 
This research has made use of NASA's Astrophysics Data System Bibliographic Services, the SIMBAD database, operated at CDS, Strasbourg, France, the NASA/IPAC Extragalactic Database, operated by the Jet Propulsion Laboratory, California Institute of Technology, under contract with NASA, and the VizieR database of astronomical catalogs (Ochsenbein et al. 2000). IRAF (Image Reduction and Analysis Facility) is distributed by NOAO, which are operated by the Association of Universities for Research in Astronomy, Inc., under cooperative agreement with the NSF.

Funding for the SDSS and SDSS-II has been provided by the Alfred P. Sloan Foundation, the Participating Institutions, the NSF, the U.S. Department of Energy, NASA, the Japanese Monbukagakusho, the Max Planck Society, and the Higher Education Funding Council for England. The SDSS Web Site is http://www.sdss.org/.

The SDSS is managed by the Astrophysical Research Consortium for the Participating Institutions. The Participating Institutions are the American Museum of Natural History, Astrophysical
Institute Potsdam, University of Basel, University of Cambridge, Case Western Reserve University, University of Chicago, Drexel University, Fermilab, the Institute for Advanced Study, the Japan Participation Group, Johns Hopkins University, the Joint Institute for Nuclear Astrophysics, the Kavli Institute for Particle Astrophysics and Cosmology, the Korean Scientist Group, the Chinese Academy of Sciences (LAMOST), Los Alamos National Laboratory, the Max-Planck-Institute for Astronomy (MPIA), the Max-Planck-Institute for Astrophysics (MPA), New Mexico State University, Ohio State University, University of Pittsburgh, University of Portsmouth, Princeton University, the United States Naval Observatory, and the University of Washington.

The Two Micron All Sky Survey was a joint project of the University of Massachusetts and the Infrared Processing and Analysis Center (California Institute of Technology). The University of Massachusetts was responsible for the overall management of the project, the observing facilities, and the data acquisition. The Infrared Processing and Analysis Center was responsible for data processing, distribution, and archiving.

\section{APPENDIX A}

\section{ChaMP SOURCES WITH SIMBAD COUNTERPARTS}

In Table 5 we present the optical data for the 66 stars cataloged in SIMBAD that we have identified as ChaMP X-ray sources, and include additional information (spectral type, binarity, variability) where available. We searched the literature for evidence that these stars had been identified as X-ray sources and could find no previous X-ray detections; we therefore consider these all to be new X-ray source identifications. Four stars are positionally coincident with X-ray sources in other Chandra catalogs but are not explicitly listed in SIMBAD as X-ray emitters or identified in these catalogs as stars, and we therefore consider them also to be new identifications. CXOMP J084944.7+445840 is among the sources detected in Lynx (Stern et al. 2002) and listed in the Serendipitous Extragalactic X-Ray Source Identification (SEXSI; Harrison et al. 2003) catalog but is unidentified in both catalogs. CXOMP J085005.3+445819 and J090941.7+541939 are both unidentified SEXSI sources. Finally, CXOMP J162157.2+381734 is less than 10" from 1RXS J162157.6+ 381727, an unidentified RASS source.

Thirteen ChaMP stellar sources do not have SIMBAD optical counterparts but are included in other X-ray catalogs. However, our examination of these catalogs reveals no additional information about the nature of these sources, and we also consider these to be new X-ray-source identifications. For example, CXOMP J084854.0+450230 is within 1" of the X-ray source [STS2002] 43 (Stern et al. 2002), but the catalog for that survey does not include an identification for this X-ray source or for two other ChaMP sources. Similarly, eight ChaMP sources listed in the SEXSI catalog and two observed in Bootes by Wang et al. (2004) are not identified. CXOMP J141120.7+521411 is included in three catalogs and unidentified in all three, although a magnitude is given for the counterpart by Zickgraf et al. (2003) in their catalog of RASS BSC sources. CXOMP J125152.2+000528 is listed by Zickgraf et al. (2003) but is unidentified. CXOMP J214229.3+123322 is within $4^{\prime \prime}$ of the unidentified RASS source 1RXS J214229.5+123323. These sources are listed in Table 6. In total, we have 79 ChESS stars with cataloged optical or X-ray data but which had not previously been identified as stellar X-ray sources.

Finally, 10 ChESS stars are previously known stellar X-ray sources. We list these in Table 7. A full examination of the properties of these stars (e.g., a comparison of their previously reported fluxes to those detected by Chandra) is beyond the scope of this paper.

TABLE 5

ChaMP Sources with SIMBAD Counterparts

\begin{tabular}{|c|c|c|c|c|c|}
\hline CXOMP & $\begin{array}{l}\text { SIMBAD } \\
\text { Counterpart }\end{array}$ & $\begin{array}{l}\text { Sep. } \\
(\operatorname{arcsec})\end{array}$ & $\begin{array}{c}B \\
(\mathrm{mag})\end{array}$ & $\begin{array}{c}V \\
(\mathrm{mag})\end{array}$ & Comments \\
\hline J015941.6-084506.. & BD-09 375 & 0.4 & 11.90 & 10.80 & \\
\hline J015959.7+003220 ... & [BHR2005] 829-29 & 1.8 & . & $\ldots$ & M3.5 V \\
\hline J023132.5-072724.. & TYC $4704-81-1$ & 1.1 & 11.70 & 11.20 & \\
\hline J025951.7+004619.. & [BHR2005] 832-7 & 0.0 & . & $\ldots$ & M5.5 V \\
\hline $\mathrm{J} 072501.4+371351 \ldots$ & TYC $2464-396-1$ & 0.9 & 10.55 & 9.52 & \\
\hline J072545.4+365905 .... & $\mathrm{BD}+371715$ & 0.6 & 10.72 & 10.28 & F5 \\
\hline 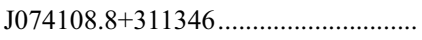 & 2MASS J07410881+3113463 & 0.0 & 19.20 & $\ldots$ & $\ldots$ \\
\hline 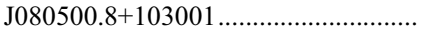 & HD 66686 & 1.9 & 8.17 & 7.29 & G5 III \\
\hline 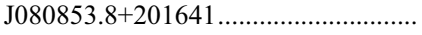 & BD+20 2009 & 2.3 & 10.86 & 10.60 & F5 \\
\hline $\mathrm{J} 080920.5+202322 .$. & BD+20 2011 & 1.2 & 10.82 & 10.14 & F0 \\
\hline $\mathrm{J} 081539.2+364742 .$. & TYC $2482-1192-1$ & 1.7 & 11.70 & 11.10 & .. \\
\hline 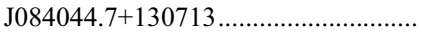 & TYC $805-471-1$ & 1.2 & 11.80 & 11.20 & .. \\
\hline $\mathrm{J} 084944.7+445840^{\mathrm{a}, \mathrm{b}} \ldots \ldots \ldots \ldots \ldots$ & HD 75117 & 0.8 & 8.45 & 7.98 & G0 \\
\hline
\end{tabular}


TABLE 5-Continued

\begin{tabular}{|c|c|c|c|c|c|}
\hline CXOMP & $\begin{array}{l}\text { SIMBAD } \\
\text { Counterpart }\end{array}$ & $\begin{array}{c}\text { Sep. } \\
(\operatorname{arcsec})\end{array}$ & $\begin{array}{c}B \\
(\mathrm{mag})\end{array}$ & $\begin{array}{c}V \\
(\mathrm{mag})\end{array}$ & Comments \\
\hline $\mathrm{J} 085005.3+445819^{\mathrm{b}}$ & HD 75172 & 4.2 & 9.24 & 8.98 & F5 \\
\hline 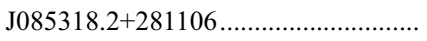 & TYC 1949-1327-1 & 1.5 & 11.20 & 10.39 & $\ldots$ \\
\hline J085325.7+232919... & 2MASS J08532577+2329194 & 1.1 & $\ldots$ & $\ldots$ & $\ldots$ \\
\hline J085711.0+085651.. & TYC 811-1921-1 & 2.6 & 11.10 & 10.70 & $\ldots$ \\
\hline $\mathrm{J} 090941.7+541939^{\mathrm{b}}$. & TYC $3805-167-1$ & 2.5 & 10.95 & 10.50 & $\ldots$ \\
\hline J091432.7+561238.. & HD 237796 & 0.6 & 10.65 & 9.61 & K5 \\
\hline J091444.7+562104 & TYC 3809-904-1 & 1.8 & 11.40 & 10.90 & $\ldots$ \\
\hline 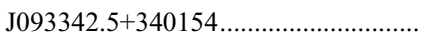 & TYC $2497-1154-1$ & 0.8 & 11.70 & 11.30 & $\ldots$ \\
\hline J093905.4+005146 & TYC $235-1240-1$ & 2.1 & 11.50 & 11.00 & $\ldots$ \\
\hline J095427.2+410515 & $\mathrm{BD}+412023$ & 1.7 & 11.10 & 10.36 & K0 \\
\hline 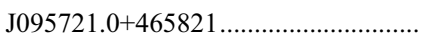 & TYC $3433-1205-1$ & 2.0 & 11.60 & 10.90 & $\ldots$ \\
\hline 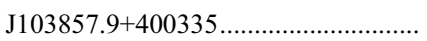 & TYC $3005-806-1$ & 1.3 & 10.50 & 10.22 & $\ldots$ \\
\hline 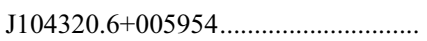 & TYC $254-1148-1$ & 3.3 & 11.90 & 11.10 & $\ldots$ \\
\hline $\mathrm{J} 105202.9+160544 \ldots \ldots \ldots \ldots \ldots \ldots \ldots \ldots \ldots$ & CCDM J10520+1606AB & 0.6 & 8.68 & 8.31 & $\mathrm{~F} 5$ ** \\
\hline 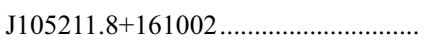 & $\mathrm{BD}+162181$ & 0.9 & 9.93 & 9.66 & F5 \\
\hline $\mathrm{J} 111446.9+532038 \ldots \ldots \ldots \ldots \ldots \ldots \ldots \ldots \ldots$ & TYC $3824-287-1$ & 1.2 & 11.19 & 10.71 & $\ldots$ \\
\hline 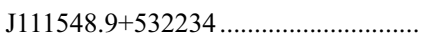 & 2MASS J11154905+5322345 & 1.4 & 12.80 & $\ldots$ & $\ldots$ \\
\hline $\mathrm{J} 111607.3+013512 .$. & CCDM J11161+0135AB & 0.8 & 9.48 & 9.01 & $\mathrm{~F} 8^{* *}$ \\
\hline 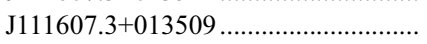 & CCDM J11161+0135AB & 3.2 & 9.48 & 9.01 & $\mathrm{~F} 8$ ** \\
\hline 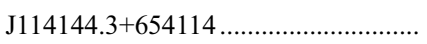 & HD 101557 & 1.5 & 9.65 & 8.97 & G5 \\
\hline 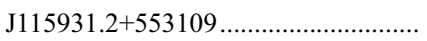 & GPM $179.879716+55.519459$ & 1.2 & 12.00 & 11.50 & $\ldots$ \\
\hline $\mathrm{J} 120041.3+290512 \ldots \ldots \ldots \ldots \ldots \ldots \ldots \ldots$ & BD+29 2244 & 4.1 & 11.08 & 10.57 & F6 \\
\hline 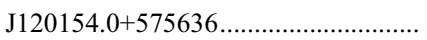 & HD 104482 & 0.9 & 9.48 & 9.02 & F5 \\
\hline $\mathrm{J} 120205.1+575539 \ldots$ & HD 238063 & 0.4 & 10.37 & 9.97 & F5 \\
\hline 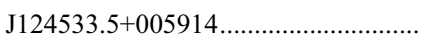 & HD 110935 & 1.5 & 9.98 & 9.08 & G5 \\
\hline $\mathrm{J} 125152.0+000505 \ldots \ldots \ldots \ldots \ldots \ldots \ldots \ldots \ldots \ldots$ & HD 111816 & 1.0 & 8.31 & 7.83 & F8 \\
\hline $\mathrm{J} 125605.1+260117 \ldots \ldots \ldots \ldots \ldots \ldots \ldots \ldots$ & $\mathrm{BD}+262407$ & 2.3 & 10.09 & 9.73 & F2 \\
\hline $\mathrm{J} 125615.7+564817 \ldots$ & GSC $03845-00748$ & 1.2 & 12.50 & $\ldots$ & $\ldots$ \\
\hline 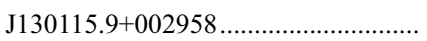 & NLTT 32614 & 2.6 & 10.45 & 9.59 & K0 PM* \\
\hline 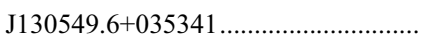 & LSPM J1305+0353 & 0.4 & 18.80 & & $\mathrm{PM}^{*}$ \\
\hline 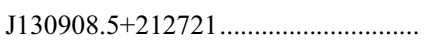 & CCDM J13091+2127AB & 0.9 & 8.93 & 8.34 & $\mathrm{G} 5{ }^{* *}$ \\
\hline $\mathrm{J} 130955.3+573403 \ldots \ldots \ldots \ldots \ldots \ldots \ldots \ldots \ldots$ & HD 114505 & 1.1 & 9.80 & 9.21 & G \\
\hline J131043.9-031731 ……............................ & HD 114465 & 0.7 & 9.12 & 8.71 & F8 \\
\hline $\mathrm{J} 131057.7+011553 \ldots$ & StKM $1-1052$ & 3.2 & & 11.40 & K5 \\
\hline $\mathrm{J} 133143.4+111132 .$. & $\mathrm{BD}+112580$ & 0.7 & 11.00 & 9.96 & K0 \\
\hline 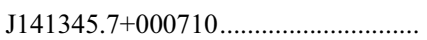 & 2MASS J14134569+0007068 & 2.8 & 18.96 & $\ldots$ & $\ldots$ \\
\hline $\mathrm{J} 141349.2+000806 \ldots \ldots \ldots \ldots \ldots \ldots \ldots \ldots \ldots$ & 2MASS J14134944+0008055 & 3.5 & 15.97 & $\ldots$ & $\ldots$ \\
\hline $\mathrm{J} 141808.1+264743 \ldots \ldots \ldots \ldots \ldots \ldots \ldots \ldots \ldots$ & HD 125320 & 0.8 & 8.93 & 8.19 & G5 IV \\
\hline 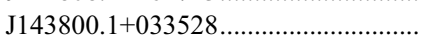 & HD 128645 & 0.5 & 9.72 & 9.40 & F5 \\
\hline $\mathrm{J} 144232.8+011710 \ldots \ldots \ldots \ldots \ldots \ldots \ldots$ & $\mathrm{BD}+012965$ & 0.7 & 10.41 & 9.10 & K0 III \\
\hline $\mathrm{J} 144300.8+012423 \ldots \ldots \ldots \ldots \ldots \ldots \ldots \ldots$ & 2MASS J14430071+0124239 & 1.8 & 15.30 & & \\
\hline $\mathrm{J} 144430.1-012826 \ldots \ldots \ldots \ldots \ldots \ldots \ldots \ldots \ldots$ & 2MASS J14443011-0128261 & 0.3 & 15.30 & 15.29 & $\mathrm{RR}^{*}$ \\
\hline 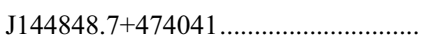 & GSC $03477-01108$ & 1.1 & 14.00 & $\ldots$ & $\ldots$ \\
\hline $\mathrm{J} 152209.8+524435 \ldots \ldots \ldots \ldots \ldots \ldots \ldots \ldots \ldots$ & HD 137146 & 1.9 & 9.70 & 9.26 & F8 \\
\hline J153203.6+240505 & $\mathrm{BD}+242880$ & 0.8 & 9.86 & 9.42 & F8 \\
\hline 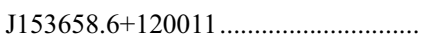 & GPM $234.244393+12.002844$ & 0.7 & 12.40 & 11.30 & \\
\hline 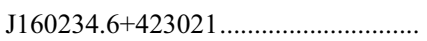 & HD 144129 & 0.6 & 9.85 & 9.46 & F5 \\
\hline $\mathrm{J} 162157.2+381734^{\mathrm{c}} \ldots \ldots \ldots \ldots \ldots \ldots \ldots \ldots$ & TYC $3062-1317-1$ & 0.2 & 11.80 & 10.50 & $\mathrm{Ce}^{* \mathrm{~d}}$ \\
\hline 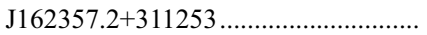 & TYC $2580-796-1$ & 0.7 & 11.70 & 11.50 & $\ldots$ \\
\hline 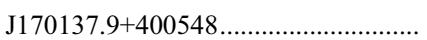 & TYC $3076-801-1$ & 3.0 & 10.93 & 10.11 & $\ldots$ \\
\hline J171952.9+263003.. & NLTT 44615 & 1.0 & 13.80 & 13.60 & $\mathrm{PM}^{*}$ \\
\hline $\mathrm{J} 231820.3+003129$.. & HD 219752 & 1.1 & 9.54 & 9.11 & F5 \\
\hline 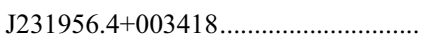 & TYC $577-673-1$ & 1.0 & 11.90 & 11.20 & $\ldots$ \\
\hline
\end{tabular}

Notes.-The double asterisks $\left({ }^{* *}\right)$ indicate a double or multiple star, "PM" ${ }^{*}$ indicates a high proper motion stars, "RR*" a RR Lyrae-type variable, and "Ce" " a Cepheid-type variable.

${ }^{a}$ Source cataloged in Stern et al. (2002).

b Source cataloged in Harrison et al. (2003).

c Source cataloged in Vizier Online Data Catalog, 9029 (W. Voges et al. 2000).

d While this star is classified as a Cepheid by Akerlof et al. (2000), inspection of the Northern Sky Variability Survey (Woźniak et al. 2004) light curve for this object suggests that this is not a classical Cepheid. 
TABLE 6

ChaMP Sources Included in Other X-Ray Catalogs

\begin{tabular}{|c|c|c|c|}
\hline CXOMP & Other Name & $\begin{array}{c}\text { Sep. } \\
(\operatorname{arcsec})\end{array}$ & Catalog \\
\hline \multirow{2}{*}{ J084854.0+450230 ......................... } & [STS2002] 43 & 1.1 & Stern et al. (2002) \\
\hline & CXOSEXSI J084854.0+450231 & 1.4 & Harrison et al. (2003) \\
\hline J084913.8+444758.. & [STS2002] 88 & 1.8 & Stern et al. (2002) \\
\hline \multirow[t]{2}{*}{ 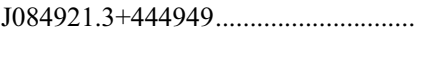 } & CXOSEXSI J084921.2+444948 & 0.5 & Harrison et al. (2003) \\
\hline & [STS2002] 106 & 0.9 & Stern et al. (2002) \\
\hline J091045.7+542019 ... & CXOSEXSI J091045.7+542019 & 2.9 & Harrison et al. (2003) \\
\hline 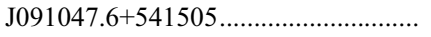 & CXOSEXSI J091047.6+541505 & 0.8 & Harrison et al. (2003) \\
\hline 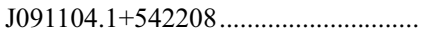 & CXOSEXSI J091104.2+542206 & 1.9 & Harrison et al. (2003) \\
\hline J115903.8+291747.. & CXOSEXSI J115903.7+291746 & 1.3 & Harrison et al. (2003) \\
\hline $\mathrm{J} 125152.2+000528 \ldots$ & [ZEH2003] RX J1251.8+0005 1 & 3.2 & Zickgraf et al. (2003) \\
\hline \multirow[t]{3}{*}{ 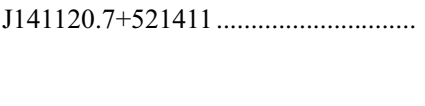 } & CXOSEXSI J141120.7+521411 & 0.2 & Harrison et al. (2003) \\
\hline & [CME2001] 3C 29512 & 1.0 & Cappi et al. (2001) \\
\hline & [ZEH2003] RX J1411.3+5212 1 & 1.4 & $B=14.1 ;$ Zickgraf et al. (2003) \\
\hline 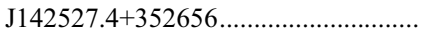 & CXOLALA1 J142527.5+352656 & 1.6 & Wang et al. (2004) \\
\hline $\mathrm{J} 142547.1+353954 \ldots \ldots \ldots \ldots \ldots \ldots \ldots \ldots \ldots$ & CXOLALA1 J142547.1+353954 & 0.9 & Wang et al. (2004) \\
\hline 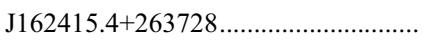 & CXOSEXSI J162415.4+263729 & 1.2 & Harrison et al. (2003) \\
\hline $\mathrm{J} 214229.3+123322$. & 1RXS J214229.5+123323 & 3.3 & Vizier Online Data Catalog, 9029 (W. Voges et al. 2000) \\
\hline
\end{tabular}

TABLE 7

Previously Known Stellar X-ray Sources with ChaMP Detections

\begin{tabular}{|c|c|c|c|c|c|}
\hline CXOMP & SIMBAD Counterpart & $\begin{array}{c}\text { Sep. } \\
(\operatorname{arcsec})\end{array}$ & $\begin{array}{c}B \\
(\mathrm{mag})\end{array}$ & $\begin{array}{c}V \\
(\mathrm{mag})\end{array}$ & Comments \\
\hline $\mathrm{J} 080813.5+210608 \ldots$ & LHS 5134 & 0.5 & 12.65 & 11.20 & M2.5 PM*; Hünsch et al. (1999) \\
\hline $\mathrm{J} 100734.8+130144 \ldots \ldots \ldots \ldots \ldots \ldots$ & NLTT 23457 & 3.6 & 8.98 & 8.40 & F8 PM*; Stocke et al. (1991) \\
\hline J105336.4+573800 .................. & TYC $3829-162-1$ & 0.6 & 11.00 & 10.43 & Ishisaki et al. $(2001)^{\mathrm{a}}$ \\
\hline J105410.3+573038 ….............. & RDS $20 \mathrm{C}$ & 0.8 & $\ldots$ & $\ldots$ & M5V; Ishisaki et al. (2001) \\
\hline J122156.1+271834 ................... & HD 107611 & 0.9 & 8.95 & 8.50 & F6V *iC; Randich et al. (1996) \\
\hline 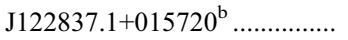 & GSC $00282-00187$ & 3.6 & & & M2; Pflueger et al. (1996) \\
\hline J125533.7+255331 ….............. & PN G339.9+88.4 & 0.8 & 9.65 & 8.86 & G5III PN; Apparao et al. (1992) \\
\hline $\mathrm{J} 134513.3+555244 \ldots \ldots \ldots \ldots \ldots \ldots$ & NLTT 35142 & 1.8 & 6.97 & 6.50 & F7IV-V PM*; Schmitt et al. (1985) \\
\hline $\mathrm{J} 135608.7+183039 \ldots \ldots \ldots \ldots \ldots \ldots$ & GSC $01470-00791$ & 1.4 & $\ldots$ & 15.00 & K5; Mason et al. (2000) \\
\hline $\mathrm{J} 171954.1+263003 \ldots \ldots \ldots \ldots \ldots \ldots$ & V* V647 Her & 1.4 & 12.98 & 11.42 & M4 Fl*; Harris \& Johnson (1985) \\
\hline
\end{tabular}

Notes.- "PM" ${ }^{*}$ indicates a high proper motion star, "*iC" a star in a cluster, "PN" a planetary nebula, "Ce $\mathrm{e}^{*}$ a Cepheid-type variable, and " $\mathrm{Fl}^{*}$ " a flare star.

${ }^{\text {a }}$ Both TYC 3829-162-1 and its probable X-ray counterpart, RX J105336.4+573802 (detected by Lehmann et al. 2001), are listed in Ishisaki et al. (2001). This is presumably an accounting error.

b Also included in the López-Santiago et al. (2007) sample.

Adelman-McCarthy, J. K., et al. 2007, ApJS, 172, 634

. 2008, ApJS, 175, 297

Akerlof, C., et al. 2000, AJ, 119, 1901

Aldcroft, T. L., Karovska, M., Cresitello-Dittmar, M. L., Cameron, R. A., \& Markevitch, M. L. 2000, Proc. SPIE, 4012, 650

Alexander, D. M., et al. 2003, AJ, 126, 539

Amado, P. J. 2003, A\&A, 404, 631

Amado, P. J., \& Byrne, P. B. 1997, A\&A, 319, 967

Anderson, S. F., et al. 2007, AJ, 133, 313

Apparao, K. M. V., Berthiaume, G. D., \& Nousek, J. A. 1992, ApJ, 397, 534

Bade, N., et al. 1998, A\&AS, 127, 145

Barkhouse, W. A., et al. 2006, ApJ, 645, 955

Bochanski, J. J., West, A. A., Hawley, S. L., \& Covey, K. R. 2007, AJ, 133, 531

Brandt, W. N., \& Hasinger, G. 2005, ARA\&A, 43, 827

Cappi, M., et al. 2001, ApJ, 548, 624

Collier Cameron, A., \& Jianke, L. 1994, MNRAS, 269, 1099

Covey, K. R., et al. 2007, AJ, 134, 2398

Cram, L. E. 1982, ApJ, 253, 768

Cutri, R. M., et al. 2003, The 2MASS All Sky Catalog of Point Sources (Pasadena: NASA/IPAC Infrared Science Archive), http://irsa.ipac.caltech.edu/ applications/Gator/

Della Ceca, R., et al. 2004, A\&A, 428, 383

Favata, F., Micela, G., Sciortino, S., \& Vaiana, G. S. 1992, A\&A, 256, 86

Feigelson, E. D., et al. 2004, ApJ, 611, 1107

\section{REFERENCES}

Freyberg, M. J., Altieri, B., Bermejo, D., Esquej, M. P., Lazaro, V., Read, A. M., \& Saxton, R. D. 2006, in The X-ray Universe 2005, ed. A. Wilson (ESA SP-604; Noordwijk: ESA), 913

Fukugita, M., et al. 1996, AJ, 111, 1748

Gehrels, N. 1986, ApJ, 303, 336

Gioia, I. M., et al. 1984, ApJ, 283, 495 1990, ApJS, 72, 567

Girardi, L., et al. 2005, A\&A, 436, 895

Green, P. J., et al. 2004, ApJS, 150, 43

Gunn, J. E., et al. 1998, AJ, 116, 3040 2006, AJ, 131, 2332

Hagen, H.-J., et al. 1995, A\&AS, 111, 195

Harris, D. E., \& Johnson, H. M. 1985, ApJ, 294, 649

Harrison, F. A., Eckart, M. E., Mao, P. H., Helfand, D. J., \& Stern, D. 2003, ApJ, 596, 944

Herbst, W., \& Miller, J. R. 1989, AJ, 97, 891

Hogg, D. W., et al. 2001, AJ, 122, 2129

Houdebine, E. R., Mathioudakis, M., Doyle, J. G., \& Foing, B. H. 1996, A\&A, 305, 209

Houdebine, E. R., \& Stempels, H. C. 1997, A\&A, 326, 1143

Hünsch, M., Schmitt, J. H. M. M., Sterzik, M. F., \& Voges, W. 1999, A\&AS, 135,319

Ishisaki, Y., Ueda, Y., Yamashita, A., Ohashi, T., Lehmann, I., \& Hasinger, G. 2001, PASJ, 53, 445 
Ivezić, Ž., et al. 2004, Astron. Nachr., 325, 583

James, D. J., Jardine, M. M., Jeffries, R. D., Randich, S., Collier Cameron, A., \& Ferreira, M. 2000, MNRAS, 318, 1217

Jardine, M. 2004, A\&A, 414, L5

Karaali, S., Bilir, S., \& Tunçel, S. 2005, Publ. Astron. Soc. Australia, 22, 24

Kim, D.-W., et al. 2004a, ApJ, 600, 59 2004b, ApJS, 150, 19 2006, ApJ, 644, 829

Kim, M., Wilkes, B. J., Kim, D.-W., Green, P. J., Barkhouse, W. A., Lee, M. G., Silverman, J. D., \& Tananbaum, H. D. 2007a, ApJ, 659, 29

Kim, M., et al. 2007b, ApJS, 169, 401

Kraus, A. L., \& Hillenbrand, L. A. 2007, AJ, 134, 2340

Lavalley, M., Isobe, T., \& Feigelson, E. 1992, in ASP Conf. Ser. 25, Astronomical Data Analysis Software and Systems I, ed. D. M. Worrall, C. Biemesderfer, \& J. Barnes (San Francisco: ASP), 245

Leggett, S. K. 1992, ApJS, 82, 351

Lehmann, I., et al. 2001, A\&A, 371, 833

López-Santiago, J., Micela, G., Sciortino, S., Favata, F., Caccianiga, A., Della Ceca, R., Severgnini, P., \& Braito, V. 2007, A\&A, 463, 165

Mason, K. O., et al. 2000, MNRAS, 311, 456

Mathioudakis, M., \& Doyle, J. G. 1989, A\&A, 224, 179

Monet, D. G., et al. 2003, AJ, 125, 984

Nieto-Santisteban, M. A., et al. 2004, in ASP Conf. Ser. 314, Astronomical Data Analysis Software and Systems (ADASS) XIII, ed. F. Ochsenbein, M. G. Allen, \& D. Egret (San Francisco: ASP), 666

Ochsenbein, F., Bauer, P., \& Marcout, J. 2000, A\&AS, 143, 23

Parejko, J. K., Constantin, A., Vogeley, M. S., \& Hoyle, F. 2008, AJ, 135, 10

Pettersen, B. R. 1987, Vistas Astron., 30, 41

Pflueger, B., Otterbein, K., \& Staubert, R. 1996, A\&A, 305, 699

Popesso, P., et al. 2004, A\&A, 423, 449

Randich, S., Schmitt, J. H. M. M., \& Prosser, C. 1996, A\&A, 313, 815

Reid, N., Hawley, S. L., \& Mateo, M. 1995, MNRAS, 272, 828

Richards, G. T., et al. 2004, ApJS, 155, 257 2006, AJ, 131, 2766

2007, in BAAS, AAS Meeting 211, 142.02

Saxton, R. D., Read, A. M., Esquej, P., Freyberg, M. J., Altieri, B., \& Bermejo, D. $2008, A \& A, 480,611$

Schmitt, J. H. M. M., Golub, L., Harnden, Jr., F. R., Maxson, C. W., Rosner, R., \& Vaiana, G. S. 1985, ApJ, 290, 307
Schmitt, J. H. M. M., \& Liefke, C. 2004, A\&A, 417, 651

Schmitt, J. H. M. M., et al. 1995, ApJ, 450, 392

Scranton, R., et al. 2002, ApJ, 579, 48

Silverman, J. D., et al. 2005, ApJ, 624, 630 2008, ApJ, 679, 118

Silvestri, N. M., et al. 2006, AJ, 131, 1674

Skrutskie, M. F., et al. 1997, in The Impact of Large Scale Near-IR Sky Surveys, ed. F. Garzon et al. (Dordrecht: Kluwer), 25 2006, AJ, 131, 1163

Smith, J. A., et al. 2002, AJ, 123, 2121

Smolčić, V., et al. 2004, ApJ, 615, L141

Stauffer, J. R., Caillault, J.-P., Gagne, M., Prosser, C. F., \& Hartmann, L. W. 1994, ApJS, 91, 625

Stauffer, J. R., \& Hartmann, L. W. 1986, ApJS, 61, 531

Stern, D., et al. 2002, AJ, 123, 2223

Stocke, J. T., et al. 1983, ApJ, 273, 458 1991, ApJS, 76, 813

Stoughton, C., et al. 2002, AJ, 123, 485

Sung, H., Bessell, M. S., Lee, B.-W., \& Lee, S.-G. 2002, AJ, 123, 290

Szkody, P., et al. 2003, AJ, 126, 1499 2005, AJ, 129, 2386

Vilhu, O. 1987, in Cool Stars, Stellar Systems and the Sun, ed. J. L. Linsky \& R. E. Stencel (Berlin: Springer), 110

Vilhu, O., \& Rucinski, S. M. 1983, A\&A, 127, 5

Voges, W., et al. 1999, A\&A, 349, 389

Walkowicz, L. M., Hawley, S. L., \& West, A. A. 2004, PASP, 116, 1105

Wang, J. X., et al. 2004, AJ, 127, 213

Watson, M., \& the XMM-Newton Survey Science Centre Consortium. 2006, BAAS, 38,365

Weinstein, M. A., et al. 2004, ApJS, 155, 243

Weisskopf, M. C., Brinkman, B., Canizares, C., Garmire, G., Murray, S., \& Van Speybroeck, L. P. 2002, PASP, 114, 1

West, A. A., Hawley, S. L., Bochanski, J. J., Covey, K. R., Reid, I. N., Dhital,

S., Hilton, E. J., \& Masuda, M. 2008, AJ, 135, 785

Woudt, P. A., et al. 2004, MNRAS, 351, 1015

Woźniak, P. R., et al. 2004, AJ, 127, 2436

Zickgraf, F.-J., et al. 2003, A\&A, 406, 535 